DOI 10.4171/JEMS/354

Daniele Arcara · Aaron Bertram

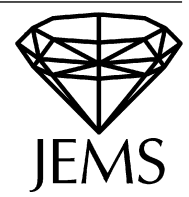

\title{
Bridgeland-stable moduli spaces for $K$-trivial surfaces
}

with an appendix by Max Lieblich

Received April 25, 2009 and in revised form May 30, 2011

\begin{abstract}
We give a one-parameter family of Bridgeland stability conditions on the derived category of a smooth projective complex surface $S$ and describe "wall-crossing behavior" for objects with the same invariants as $\mathcal{O}_{C}(H)$ when $H$ generates $\operatorname{Pic}(S)$ and $C \in|H|$. If, in addition, $S$ is a K3 or Abelian surface, we use this description to construct a sequence of fine moduli spaces of Bridgeland-stable objects via Mukai flops and generalized elementary modifications of the universal coherent sheaf. We also discover a natural generalization of Thaddeus' stable pairs for curves embedded in the moduli spaces.
\end{abstract}

\section{Contents}

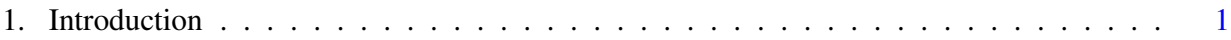

2. Stability conditions on the derived category of a surface . . . . . . . . . . . 4

3. Some Bridgeland-stable objects . . . . . . . . . . . . . . . . . . . . . 8

4. Families and walls . . . . . . . . . . . . . . . . . . . . . . . 13

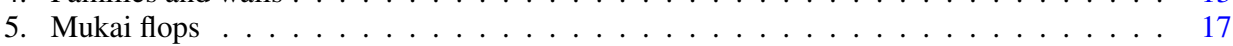

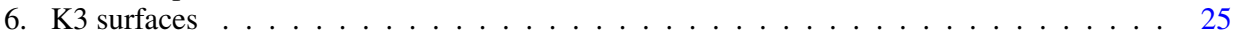

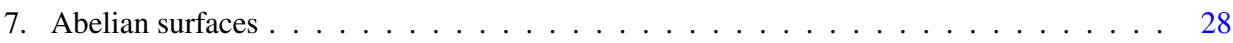

8. Stable pairs . . . . . . . . . . . . . . . . . . . . . . 29

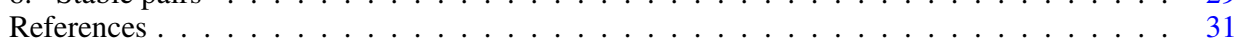

Appendix. The openness of tilted hearts (by Max Lieblich) $\ldots \ldots \ldots \ldots$. . . . . . . 32

References . . . . . . . . . . . . . . . . . . . . . . . 37

\section{Introduction}

Let $X$ be a smooth complex projective variety of dimension $n$. An ample divisor class $H$ on $X$ defines a slope function on torsion-free sheaves $E$ on $X$ via

$$
\mu_{H}(E)=\left(\int_{X} c_{1}(E) \cdot H^{n-1}\right) / \operatorname{rk}(E) .
$$

D. Arcara: Department of Mathematics, Saint Vincent College, 300 Fraser Purchase Road, Latrobe, PA 15650-2690, USA; e-mail: daniele.arcara@email.stvincent.edu

A. Bertram: Department of Mathematics, University of Utah, 155 S. 1400 E., Room 233, Salt Lake City, UT 84112-0090, USA; e-mail: bertram@math.utah.edu

M. Lieblich: Department of Mathematics, University of Washington, Box 354350, Seattle, WA 98195-4350, USA; e-mail: lieblich@math.washington.edu 
This slope function is a measure of the growth of the Hilbert function of $E$, but it also allows one to define the important notion of $H$-stability:

Definition. $E$ is $H$-stable if $\mu_{H}(F)<\mu_{H}(E)$ for all $F \subset E$ with $\operatorname{rk}(F)<\operatorname{rk}(E)$.

It is well-known that this notion allows one to classify the torsion-free sheaves on $X$ via:

- moduli for $H$-stable torsion-free sheaves with fixed Hilbert polynomial,

- Jordan-Hölder filtrations for $H$-semistable torsion-free sheaves, and

- Harder-Narasimhan filtrations for arbitrary torsion-free sheaves.

Bridgeland slope function, in contrast, is defined on the (bounded) derived category $\mathcal{D}(X)$ of coherent sheaves on $X$. It is a pair $\left(Z, \mathcal{A}^{\#}\right)$ consisting of a linear central charge

$$
Z: K(\mathcal{D}(X)) \rightarrow \mathbb{C}
$$

on the Grothendieck group, together with the heart $\mathcal{A}^{\#}$ of a t-structure on $\mathcal{D}(X)$ that is compatible with the central charge in the sense that

$$
Z(A) \in \mathcal{H}=\left\{\rho e^{i \phi} \mid \rho>0,0<\phi \leq \pi\right\}
$$

for all non-zero objects $A$ of $\mathcal{A}^{\#}$. This allows one to define a (possibly infinite-valued) slope

$$
\mu_{Z}(A):=-\frac{\operatorname{Re}(Z(A))}{\operatorname{Im}(Z(A))}
$$

for objects of $\mathcal{A}^{\#}$ analogous to the $H$-slope on coherent sheaves. The pair $\left(Z, \mathcal{A}^{\#}\right)$ is called a Bridgeland stability condition if arbitrary objects of $\mathcal{A}^{\#}$ have finite Harder-Narasimhan filtrations with respect to the slope $\mu_{Z}$ (see [Bri07] for more details).

In this paper, we will consider central charges of the form

$$
Z(E)=-\int_{S} e^{-(D+i F)} \operatorname{ch}(E) \quad \text { and } \quad Z^{\prime}(E)=-\int_{S} e^{-(D+i F)} \operatorname{ch}(E) \sqrt{\operatorname{td}(S)}
$$

on a smooth projective surface $S$, where $F$ is an ample $\mathbb{R}$-divisor, and $D$ is an arbitrary $\mathbb{R}$-divisor. Following Bridgeland's argument for K3 surfaces [Bri08], we show that the former always has a natural partner $t$-structure $\mathcal{A}^{\#}$ (depending upon $D$ and the ray generated by $F$ ) such that the pair $\left(Z, \mathcal{A}^{\#}\right)$ defines a stability condition. Our main results focus further on the one-parameter family of stability conditions on a fixed abelian category $\mathcal{A}^{\#}$, where

$$
\operatorname{Pic}(S)=\mathbb{Z}[H], \quad D=\frac{1}{2} H, \quad \text { and } \quad F=t H, \quad t>0,
$$

This family of stability conditions is well-tuned to study the stability of objects $E \in \mathcal{A}^{\#}$ with Chern class invariants

$$
\operatorname{ch}(E)=H+H^{2} / 2=\operatorname{ch}\left(\mathcal{O}_{S}(H)\right)-\operatorname{ch}\left(\mathcal{O}_{S}\right)
$$


in the sense that we will be able to state the precise set of stable objects (depending on $t$ ) with those invariants. Moreover, in the $K$-trivial case (i.e. when $S$ is a K3 or Abelian surface), we will use this knowledge to construct proper moduli spaces of Bridgeland-stable objects by starting with the relative Jacobian (the moduli of stable objects for $t \gg 0$ ) and performing a sequence of Mukai flops as $t$ passes over a series of "walls." This in particular exhibits a sequence of birational models of the relative Jacobian, which seem to be new, although they encode quite a lot of interesting results on the positivity of the line bundle $\mathcal{O}_{S}(H)$ on the surface.

To get an idea of the wall-crossing phenomenon, consider the exact sequence of coherent sheaves

$$
0 \rightarrow \mathcal{O}_{S} \rightarrow \mathcal{O}_{S}(H) \rightarrow i_{*} \mathcal{O}_{C}(H) \rightarrow 0
$$

for some curve $C \in|H|$. This, it turns out, will not be an exact sequence of objects in our category $\mathcal{A}^{\#}$. Rather, the sequence

$$
0 \rightarrow \mathcal{O}_{S}(H) \rightarrow i_{*} \mathcal{O}_{C}(H) \rightarrow \mathcal{O}_{S}[1] \rightarrow 0
$$

coming from the "turned" distinguished triangle in $\mathcal{D}(S)$ will be a short exact sequence of objects of $\mathcal{A}^{\#}$. Below the critical "wall" value $t=1 / 2$, we will have $\mu_{Z}\left(\mathcal{O}_{S}(H)\right)>$ $\mu_{Z}\left(i_{*} \mathcal{O}_{C}(H)\right)$, exhibiting $i_{*} \mathcal{O}_{C}(H)$ as an unstable object of $\mathcal{A}^{\#}(!)$. The "replacement" stable object(s) will be of the form

$$
0 \rightarrow \mathcal{O}_{S}[1] \rightarrow E \rightarrow \mathcal{O}_{S}(H) \rightarrow 0
$$

which are parametrized by $\mathbb{P}\left(\operatorname{Ext}_{\mathcal{A}^{\#}}^{1}\left(\mathcal{O}_{S}(H), \mathcal{O}_{S}[1]\right)\right)=\mathbb{P}\left(\operatorname{Ext}_{S}^{2}\left(\mathcal{O}_{S}(H), \mathcal{O}_{S}\right)\right) \cong$ $\mathbb{P}\left(H^{0}\left(S, \mathcal{O}_{S}(H)\right)^{*}\right)$ via Serre duality.

Our moduli functor is based upon the generalized notion of a flat family we learned from Abramovich and Polishchuk [AP06]. One would, of course, like to have an a priori construction of moduli spaces of Bridgeland-stable objects via some sort of invariant theory argument, but the fact that we are not working exclusively with coherent sheaves makes it difficult to see how to make such a construction. Instead, we rely on the fact that an Artin stack of flat families of objects of $\mathcal{A}^{\#}$ exists, using a result of Max Lieblich, which we attach as an appendix, and then work rather hard to show that stability is an open condition in the cases of interest to us (recent work by Toda [Tod07] gives an alternative, and more general, approach). We then work by induction, starting with the universal family over the relative Jacobian and elementary modifications across the Mukai flops to actually prove that each successive birationally equivalent space is indeed a fine moduli space of Bridgeland-stable objects. Thus we are able in this case to carry out the program envisioned by Bridgeland at the very end of [Bri08].

The methods introduced here should be useful in the construction of Bridgeland-stable moduli spaces of objects with other invariants on surfaces both with and without the $K$-trivial assumption. 


\section{Stability conditions on the derived category of a surface}

We start with some general remarks on the bounded derived category of coherent sheaves $\mathcal{D}(S)$ for the uninitiated reader. Derived categories were introduced by Verdier in [Ver63]. For a comprehensive introduction, see [Mil].

The objects of $\mathcal{D}(S)$ are complexes (with bounded cohomology)

$$
\cdots \rightarrow E_{i-1} \stackrel{d_{i-1}}{\rightarrow} E_{i} \stackrel{d_{i}}{\rightarrow} E_{i+1} \rightarrow \cdots
$$

of coherent sheaves, and homotopy classes of maps of complexes are maps in $\mathcal{D}(S)$. Let

$$
\mathcal{H}^{i}(E):=\operatorname{ker} d_{i} / \operatorname{im} d_{i-1}
$$

denote the cohomology sheaves of the complex. A (homotopy class of) map(s) of complexes

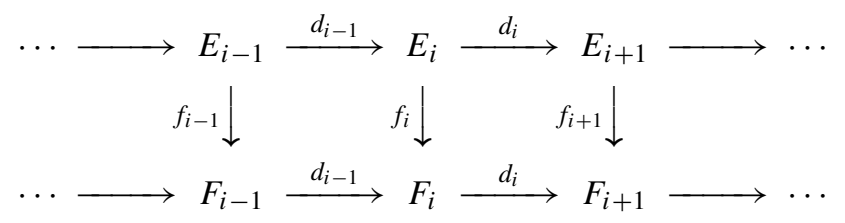

is a quasi-isomorphism if it induces isomorphisms on all cohomology sheaves. The full class of maps in $\mathcal{D}(S)$ is obtained by formally inverting all quasi-isomorphisms. Thus, in particular, quasi-isomorphic complexes represent isomorphic objects.

Here are a few facts about the derived category:

- $E[1]$ (and $f[1])$ denote the shifts: $(E[1])_{i}=E_{i+1},(f[1])_{i}=f_{i+1}$.

- $\mathcal{D}(S)$ is a triangulated category. It does not make sense to talk about kernels and cokernels of a map $f: E \rightarrow F$. Rather, the map $f$ induces a cone $C$ and a distinguished triangle

$$
\cdots \rightarrow E \stackrel{f}{\rightarrow} F \rightarrow C \rightarrow E[1] \stackrel{f[1]}{\longrightarrow} F[1] \rightarrow \cdots .
$$

- Given two coherent sheaves $E$ and $F$, there is an isomorphism

$$
\operatorname{Hom}_{\mathcal{D}(S)}(E[m], F[n]) \cong \operatorname{Ext}_{S}^{n-m}(E, F) .
$$

- A short exact sequence of sheaves

$$
0 \rightarrow K \rightarrow E \rightarrow Q \rightarrow 0
$$

induces a distinguished triangle

$$
\cdots \rightarrow K \rightarrow E \rightarrow Q \rightarrow K[1] \rightarrow E[1] \rightarrow Q[1] \rightarrow \cdots
$$

where $(Q \rightarrow K[1]) \in \operatorname{Hom}_{\mathcal{D}(S)}(Q, K[1])=\operatorname{Ext}_{S}^{1}(Q, K)$ is the extension class. 
- A distinguished triangle

$$
\cdots \rightarrow F \rightarrow E \rightarrow G \rightarrow F[1] \rightarrow \cdots
$$

induces a long exact sequence of cohomologies

$$
\cdots \rightarrow \mathcal{H}^{i}(F) \rightarrow \mathcal{H}^{i}(E) \rightarrow \mathcal{H}^{i}(G) \rightarrow \mathcal{H}^{i+1}(F) \rightarrow \cdots
$$

in the category of coherent sheaves on $S$.

- The derived dual $E^{\vee}$ of an object $E \in \mathcal{D}(S)$ is defined as $R \mathcal{H} \operatorname{com}\left(E, \mathcal{O}_{S}\right)$, where $R \mathcal{H}$ om is the derived functor induced by the $\mathcal{H}$ om functor on coherent sheaves.

- It is always true that $E^{\vee \vee}=E$ for objects of the derived category.

- If $E$ is a sheaf, the derived dual $E^{\vee}$ is represented by a complex with

$$
\mathcal{H}^{i}\left(E^{\vee}\right)=\mathcal{E} x t_{S}^{i}\left(E, \mathcal{O}_{S}\right)
$$

- The derived dual of a distinguished triangle

$$
\cdots \rightarrow F \rightarrow E \rightarrow G \rightarrow F[1] \rightarrow \cdots
$$

is the distinguished triangle

$$
\cdots \rightarrow G^{\vee} \rightarrow E^{\vee} \rightarrow F^{\vee} \rightarrow G^{\vee}[1] \rightarrow \cdots .
$$

- If three consecutive terms $F, E$, and $G$ of a distinguished triangle

$$
\cdots \rightarrow F \rightarrow E \rightarrow G \rightarrow F[1] \rightarrow \cdots
$$

are in the heart, $\mathcal{A}$, of a $t$-structure on $\mathcal{D}(S)$, then they determine a short exact sequence $0 \rightarrow F \rightarrow E \rightarrow G \rightarrow 0$ of objects in $\mathcal{A}$.

We will only be concerned here with particular sorts of $t$-structures on $\mathcal{D}(S)$ obtained by tilting. In general, tilting is obtained as follows, starting with an abelian category $\mathcal{A}$.

Definition ([HRS96]). A pair $(\mathcal{T}, \mathcal{F})$ of full subcategories in $\mathcal{A}$ is called a torsion pair if:

(TP1) $\operatorname{Hom}_{\mathcal{A}}(T, F)=0$ for every $T \in \mathcal{T}$ and $F \in \mathcal{F}$.

(TP2) Every object $E \in \mathcal{A}$ fits into a short exact sequence

$$
0 \rightarrow T \rightarrow E \rightarrow F \rightarrow 0 \text { with } T \in \mathcal{T} \text { and } F \in \mathcal{F} .
$$

Lemma ([HRS96]). Let $(\mathcal{T}, \mathcal{F})$ be a torsion pair in an abelian category $\mathcal{A}$. If $\mathcal{A}$ is the heart of a bounded $t$-structure on a triangulated category $\mathcal{D}$, then the full subcategory of $\mathcal{D}$,

$$
\mathcal{A}^{\#}=\left\{E \in \mathcal{D} \mid H^{-1}(E) \in \mathcal{F}, H^{0}(E) \in \mathcal{T}, H^{j}(E)=0 \text { for } j \neq-1,0\right\},
$$

is the heart of another $t$-structure on $\mathcal{D}$, hence in particular an abelian category. 
Remark. A short exact sequence $0 \rightarrow K \rightarrow E \rightarrow Q \rightarrow 0$ of objects of $\mathcal{A}^{\#}$ gives rise to a long exact sequence

$$
0 \rightarrow H^{-1}(K) \rightarrow H^{-1}(E) \rightarrow H^{-1}(Q) \rightarrow H^{0}(K) \rightarrow H^{0}(E) \rightarrow H^{0}(Q) \rightarrow 0
$$

of objects of $\mathcal{A}$, where $H^{-1}(K), H^{-1}(E), H^{-1}(Q) \in \mathcal{F}$ and $H^{0}(K), H^{0}(E), H^{0}(Q) \in \mathcal{T}$.

Our tilts: Given $\mathbb{R}$-divisors $D, F \in H^{1,1}(S, \mathbb{R})$ on a surface $S$, with $F$ ample, then as in $\S 1$, the $F$-slope of a torsion-free coherent sheaf $E$ on $S$ is given by

$$
\mu_{F}(E)=\left(\int_{S} c_{1}(E) \cdot F\right) / \operatorname{rk}(E)
$$

and all coherent sheaves on $S$ have a unique Harder-Narasimhan filtration

$$
E_{0} \subset E_{1} \subset \cdots \subset E_{n(E)}=E
$$

characterized by the property that $E_{0}=\operatorname{tors}(E)$ is the torsion subsheaf and each $E_{i} / E_{i-1}$ is a torsion-free $F$-semistable sheaf of slope $\mu_{i}$ (i.e. an extension of $F$-stable sheaves of slope $\mu_{i}$ ) for a strictly decreasing sequence

$$
\mu_{F-\max }(E):=\mu_{1}>\cdots>\mu_{n(E)}=: \mu_{F-\min }(E) .
$$

Definition. Let $\mathcal{A}$ be the category of coherent sheaves on $S$, let $\mathcal{T}$ be the full subcategory of coherent sheaves $E$ such that

$$
\mu_{F-\min }(E / \operatorname{tors}(E))>\int_{S} D \cdot F,
$$

and let $\mathcal{F}$ be the full subcategory of torsion-free sheaves $E$ such that

$$
\mu_{F-\max }(E) \leq \int_{S} D \cdot F \text {. }
$$

Note that this only depends upon the ray spanned by $F$. Now define $\mathcal{A}_{(D, F)}^{\#}$ by applying the Lemma to the standard $t$-structure on the bounded derived category of coherent sheaves on $S$.

Our charges: For now we will only consider the charges

$$
Z_{(D, F)}(E):=-\int_{S} e^{-(D+i F)} \operatorname{ch}(E)
$$

defined on complexes of coherent sheaves by $Z_{(D, F)}(E):=\sum(-1)^{i} Z_{(D, F)}\left(H^{i}(E)\right)$, so that in particular

$$
Z_{(D, F)}(E)=Z_{(D, F)}\left(H^{0}(E)\right)-Z_{(D, F)}\left(H^{-1}(E)\right)
$$

for objects $E$ of the category $\mathcal{A}^{\#}$.

Recall the Hodge Index Theorem and the Bogomolov-Gieseker inequality for surfaces (see, for example, [Fri98]): 
Theorem (Hodge Index). If $D$ is an $\mathbb{R}$-divisor on $S$ and $F$ is an ample $\mathbb{R}$-divisor, then

$$
D \cdot F=0 \Rightarrow D^{2} \leq 0 \text {. }
$$

Theorem (Bogomolov-Gieseker Inequality). If $E$ is an $F$-stable torsion-free sheaf on $S$, then

$$
\operatorname{ch}_{2}(E) \leq \frac{c_{1}^{2}(E)}{2 \cdot \operatorname{rk}(E)} .
$$

As an immediate corollary of these two results, we have:

Corollary 2.1. Each pair $\left(Z_{(D, F)}, \mathcal{A}_{(D, F)}^{\#}\right)$ is a Bridgeland slope function.

Proof. Since each object $E$ of $\mathcal{A}_{(D, F)}^{\#}$ fits into an exact sequence

$$
0 \rightarrow H^{-1}(E)[1] \rightarrow E \rightarrow H^{0}(E) \rightarrow 0
$$

with $H^{-1}(E) \in \mathcal{F}$ and $H^{0}(E) \in \mathcal{T}$, and since $\mathcal{H}$ is closed under addition, it suffices to show that:

(1) $Z_{(D, F)}(T) \in \mathcal{H}$ for all torsion sheaves on $S$,

(2) $Z_{(D, F)}(E) \in \mathcal{H}$ for all $F$-stable torsion-free sheaves with $\mu_{F}(E)>D \cdot F$,

(3) $Z_{(D, F)}(E[1]) \in \mathcal{H}$ for all $F$-stable torsion-free sheaves with $\mu_{F}(E) \leq D \cdot F$.

Let $Z(E):=Z_{(D, F)}(E)$ and compute

$$
Z(E)=\left(-\operatorname{ch}_{2}(E)+D \cdot c_{1}(E)-\operatorname{rk}(E)\left(D^{2} / 2-F^{2} / 2\right)\right)+i F \cdot\left(c_{1}(E)-\operatorname{rk}(E) D\right) .
$$

In (1), either $T$ is supported in dimension 1 and $\operatorname{Im}(Z(T))=c_{1}(E) \cdot F>0$ since $c_{1}(E)$ is effective, or else $T$ is supported in dimension 0 , in which case $\operatorname{Im}(Z(T))=0$, but $\operatorname{Re}(Z(T))=-\operatorname{ch}_{2}(T)<0$. So $Z(T) \in \mathcal{H}$.

$\operatorname{In}(2), \operatorname{Im}(Z(E))=F \cdot\left(c_{1}(E)-\operatorname{rk}(E) D\right)=\operatorname{rk}(E)\left(\mu_{F}(E)-D \cdot F\right)>0$. Similarly, in (3), if $\mu_{F}(E)<D \cdot H$, then $\operatorname{Im}(Z(E))<0$, so $\operatorname{Im}(Z(E[1]))>0$. Finally, if $\mu_{F}(E)=$ $D \cdot F$ and $E$ is $F$-stable, then by the Bogomolov-Gieseker inequality,

$$
\begin{aligned}
\operatorname{Re}(Z(E)) & \geq-\frac{c_{1}^{2}(E)}{2 \cdot \operatorname{rk}(E)}+D \cdot c_{1}(E)-\operatorname{rk}(E)\left(D^{2} / 2-F^{2} / 2\right) \\
& =-\frac{1}{2 \cdot \operatorname{rk}(E)}\left(\operatorname{rk}(E) D-c_{1}(E)\right)^{2}+\operatorname{rk}(E) F^{2} / 2 .
\end{aligned}
$$

But $\mu_{F}(E)=D \cdot F$ implies $\left(\operatorname{rk}(E) D-c_{1}(E)\right) \cdot F=0$, and so by the Hodge index theorem, we have $\operatorname{Re}(Z(E))>0, \operatorname{Re}(Z(E[1]))<0$, and $Z(E[1]) \in \mathcal{H}$, as desired.

Remarks. (i) In fact, each $\left(Z_{(D, F)}, \mathcal{A}_{(D, F)}^{\#}\right)$ is a Bridgeland stability condition. The existence of finite Harder-Narasimhan filtrations is immediate when $D$ and $F$ are $\mathbb{Q}$-divisors (see Proposition 7.1 of [Bri08]). In the general case of $\mathbb{R}$-divisors, the finiteness of Harder-Narasimhan filtrations is more delicate (see e.g. [BM09, §4] for a proof).

(ii) The "standard" properties of stable objects are easy to see. For example, for ( $D, F)$-stable objects $A, B \in \mathcal{A}^{\#}$, the implication

$$
\mu_{Z}(A)>\mu_{Z}(B) \Rightarrow \operatorname{Hom}_{\mathcal{A}^{\#}}(A, B)=0
$$

is immediate for Bridgeland slope functions, and if $0 \neq f \in \operatorname{Hom}_{\mathcal{A}^{\#}}(A, B)$ with $\mu_{Z}(A)$ $=\mu_{Z}(B)$, then $A \cong B$ and $f=\lambda \cdot$ id (Schur's Lemma). 


\section{Some Bridgeland-stable objects}

It is tricky, in general, to determine which objects of $\mathcal{D}(S)$ are stable for arbitrary pairs $(D, F)$. Fortunately, to determine "wall-crossing" phenomena, it is enough to consider a one-parameter family $Z_{t}:=Z_{\left(D_{t}, F_{t}\right)}$ of central charges. This is what we will do in the special case

$$
\operatorname{Pic}(S)=\mathbb{Z}[H]
$$

for the stability conditions

$$
D=\frac{1}{2} H, \quad F=t H, \quad t>0,
$$

so that if we let $Z_{t}(E)=Z_{\left(\frac{1}{2} H, t H\right)}(E)$, then

$Z_{t}(E)=-\operatorname{ch}_{2}(E)+\frac{1}{2} c_{1}(E) \cdot H+\frac{\operatorname{rk}(E) H^{2}}{2}\left(t^{2}-\frac{1}{4}\right)+i t\left(H \cdot\left(c_{1}(E)-\frac{\operatorname{rk}(E) H}{2}\right)\right)$.

The abelian category $\mathcal{A}^{\#}:=\mathcal{A}_{\left(\frac{1}{2} H, t H\right)}^{\#}$ is independent of $t$, and the $t$-stable objects of $\mathcal{A}^{\#}$ of infinite $Z_{t}$-slope are always either

(a) torsion sheaves supported in dimension zero, or

(b) shifts $E[1]$ of $H$-stable vector bundles $E$ of even rank $2 n$ with $c_{1}(E)=n H$.

All objects in $\mathcal{A}^{\#}$ of infinite slope are extensions of these. For example, if $F$ is torsion-free but not locally free of rank $2 n$ and $c_{1}(F)=n H$, let $E=F^{* *}$, and then

$$
0 \rightarrow F \rightarrow E \rightarrow T_{Z} \rightarrow 0
$$

(for a sheaf $T_{Z}$ supported on a scheme $Z$ of dimension zero) becomes the exact sequence

$$
0 \rightarrow T_{Z} \rightarrow F[1] \rightarrow E[1] \rightarrow 0
$$

exhibiting $F[1]$ as an extension in $\mathcal{A}^{\#}$ of $E[1]$ by the torsion sheaf $T_{Z}$.

Definition. An object $E$ of $\mathcal{A}^{\#}$ is $t$-stable if it is stable with respect to the central charge $Z_{t}$, i.e.

$$
\mu_{t}(K)=-\frac{\operatorname{Re}\left(Z_{t}(K)\right)}{\operatorname{Im}\left(Z_{t}(K)\right)}<\mu_{t}(E)
$$

for all subobjects $K \subset E$ in $\mathcal{A}^{\#}\left(\Leftrightarrow \mu_{t}(E)<\mu_{t}(Q)\right.$ for all surjections $E \rightarrow Q \rightarrow 0$ in $\left.\mathcal{A}^{\#}\right)$.

The following lemma establishes the $t$-stability of some basic objects of $\mathcal{A}^{\#}$.

Lemma 3.1. Let $Z \subset S$ be a subscheme of dimension zero. Then the objects

$$
\mathcal{J}_{Z}(H) \quad \text { and } \quad \mathcal{J}_{Z}^{\vee}[1]=R H o m\left(\mathcal{J}_{Z}, \mathcal{O}_{S}\right)[1]
$$

are $t$-stable for all $t>0$. 
Proof. Let $K$ be a subobject of $\mathcal{J}_{Z}(H)$ and let

$$
0 \rightarrow K \rightarrow \mathcal{J}_{Z}(H) \rightarrow Q \rightarrow 0
$$

be the associated "potentially destabilizing" sequence in $\mathcal{A}^{\#}$. This induces

$$
0 \rightarrow H^{-1}(K) \rightarrow 0 \rightarrow H^{-1}(Q) \rightarrow H^{0}(K) \rightarrow \mathcal{J}_{Z}(H) \rightarrow H^{0}(Q) \rightarrow 0,
$$

which implies that $H^{0}(Q)=\mathcal{J}_{Z}(H)$ or else $H^{0}(Q)$ is a torsion sheaf.

If $H^{0}(Q)=\mathcal{J}_{Z}(H)$, then $H^{0}(K)=H^{-1}(Q) \in \mathcal{T} \cap \mathcal{F}=0$, so $K=0$.

If $H^{0}(Q)$ is torsion, then $H^{0}(K) \in \mathcal{T}$ and $H^{-1}(Q) \in \mathcal{F}$ imply that the support of $H^{0}(Q)$ has dimension 0 , and $\operatorname{rk}\left(H^{0}(K)\right)=\operatorname{rk}\left(H^{-1}(Q)\right)+1$ and $c_{1}\left(H^{0}(K)\right)=$ $c_{1}\left(H^{-1}(Q)\right)+H$, so $\operatorname{rk}\left(H^{-1}(Q)\right)=2 n$ and $c_{1}\left(H^{-1}(Q)\right)=n H$ (or else $\left.H^{-1}(Q)=0\right)$. Thus $Q$ has infinite slope (!), and the "potentially destabilizing" sequence cannot, therefore, destabilize $\mathcal{J}_{Z}(H)$.

Turning next to $\mathcal{J}_{Z}^{\vee}[1]$, notice that

$$
\left.H^{-1}\left(\mathcal{J}_{Z}^{\vee}[1]\right)=\mathcal{O}_{S} \text { and } H^{0}\left(\mathcal{J}_{Z}^{\vee}[1]\right)=\mathcal{E} x t_{\mathcal{O}_{S}}^{2}\left(\mathcal{O}_{Z}, \mathcal{O}_{S}\right)=T \text { (torsion, supported on } Z\right)
$$

so any "potentially destabilizing" sequence

$$
0 \rightarrow K \rightarrow \mathcal{J}_{Z}^{\vee}[1] \rightarrow Q \rightarrow 0
$$

gives rise to a long exact sequence of coherent sheaves

$$
0 \rightarrow H^{-1}(K) \rightarrow \mathcal{O}_{S} \rightarrow H^{-1}(Q) \rightarrow H^{0}(K) \rightarrow T \rightarrow H^{0}(Q) \rightarrow 0 .
$$

Thus $H^{0}(Q)$ is supported in dimension 0 , and either $H^{-1}(K)=0$ or else $H^{-1}(K)=\mathcal{O}_{S}$ (otherwise the sheaf $H^{-1}(Q)$ would have torsion, which is not allowed).

If $H^{-1}(K)=\mathcal{O}_{S}$, then $c_{1}\left(H^{-1}(Q)\right)=c_{1}\left(H^{0}(K)\right)$ and $\operatorname{rk}\left(H^{-1}(Q)\right)=\operatorname{rk}\left(H^{0}(K)\right)$, which contradicts $H^{-1}(Q) \in \mathcal{F}$ and $H^{0}(K) \in \mathcal{T}$, unless of course $H^{-1}(Q)=0$. This would not destabilize $\mathcal{J}_{Z}^{\vee}[1]$, since $Q=H^{0}(Q)$ would have infinite slope, since it would be a torsion sheaf supported in dimension zero.

If $H^{-1}(K)=0$, then either $H^{-1}(Q)$ is locally free of rank $2 n$ and $c_{1}=n H$, or else $H^{-1}(Q)=\mathcal{O}_{S}$ and $H^{0}(K)$ is torsion, supported in dimension 0 . But in the first case, $H^{-1}(Q)[1]$ and $H^{0}(Q)$ have infinite slope, so $Q$ has infinite slope and $(*)$ does not destabilize. In the second case, we need to worry about $H^{0}(K)$. If $H^{0}(K) \neq 0$, then $(*)$ would destabilize $\mathcal{J}_{Z}^{\vee}[1]$ because $K=H^{0}(K) \subset \mathcal{J}_{Z}^{\vee}[1]$ would have infinite slope. But the derived dual contains no such subobjects $K$, because $\left(\mathcal{J}_{Z}^{\vee}\right)^{\vee}=\mathcal{J}_{Z}$ is a sheaf!

Remark. The object $\mathcal{J}_{Z}^{\vee}[1]$ is a surface analogue of the line bundle $\mathcal{O}_{C}(D)=I_{D}^{\vee}$ on a curve:

$$
0 \rightarrow \mathcal{O}_{C} \rightarrow \mathcal{O}_{C}(D) \rightarrow \mathcal{E} x t_{\mathcal{O}_{C}}^{1}\left(\mathcal{O}_{D}, \mathcal{O}_{C}\right) \rightarrow 0
$$

Remark. Beware of the temptation to treat $t$-stability too casually! Observe:

Lemma 3.2. The objects $\mathcal{J}_{Z}[1] \in \mathcal{A}^{\#}$ are not $t$-stable for any value of $t$. 
Proof. The exact sequence of objects in $\mathcal{A}^{\#}$,

$$
0 \rightarrow \mathcal{O}_{Z} \rightarrow \mathcal{J}_{Z}[1] \rightarrow \mathcal{O}_{S}[1] \rightarrow 0,
$$

always destabilizes $\mathcal{J}_{Z}[1]$, since $\mathcal{O}_{Z}$ has infinite slope.

Next, we turn our attention to the objects of $\mathcal{A}^{\#}$ with the invariants

$$
\operatorname{ch}(E)=0+H+H^{2} / 2 .
$$

A quick computation shows that

$$
\mu_{t}(E)=\operatorname{Re}\left(Z_{t}(E)\right)=0 \quad \text { for all } t
$$

for all $E$ with these invariants. Thus to check that $\mu_{t}(K)<\mu_{t}(E)\left(\right.$ or $\mu_{t}(K)>\mu_{t}(E)$ ) it suffices to compute the real part of $Z_{t}(K)$.

Proposition 3.3. If $E \in \mathcal{A}^{\#}$ has numerical invariants $r=0, c_{1}=H, \mathrm{ch}_{2}=H^{2} / 2$ and $E$ is $t$-stable for some value of $t$, then either $E=i_{*} L_{C}$ for some torsion-free rank-one sheaf $L_{C}$ supported on a curve $C \in|H|$, or else:

(1) $H^{0}(E)$ has torsion (if any) only in dimension 0.

(2) $H^{-1}(E)$ is locally free and $H$-stable of odd rank $2 n+1$ with $c_{1}=n H$.

(3) $H^{0}(E) / \operatorname{tors}\left(H^{0}(E)\right)$ is $H$-stable of rank $2 n+1$ with $c_{1}=(n+1) H$.

(4) The kernel of $E \rightarrow H^{0}(E) / \operatorname{tors}\left(H^{0}(E)\right)$ is the (shifted) derived dual of a torsion-free sheaf.

Proof. If $E$ is a sheaf with these invariants, then it is of the form $i_{*} L_{C}$ and any torsion in $L_{C}$ would destabilize, as all torsion sheaves on $S$ supported in dimension 0 have infinite slope. Otherwise $H^{-1}(E) \neq 0$. Now $\operatorname{rk}\left(H^{-1}(E)\right)=\operatorname{rk}\left(H^{0}(E)\right)$ and $c_{1}\left(H^{-1}(E)\right)=$ $c_{1}\left(H^{0}(E)\right)-H$ from the invariants, and this, together with $H^{-1}(E) \in \mathcal{F}$ and $H^{0}(E) \in \mathcal{T}$, forces (1). If $\operatorname{rk}\left(H^{-1}(E)\right)=2 n$, then $c_{1}\left(H^{-1}(E)\right)=n H$ is also forced, and $H^{-1}(E)[1]$ $\subset E$ would be a subobject of infinite slope, contradicting the stability of $E$ for each value of $t$. So $H^{-1}(E)$ has odd rank $2 n+1$, and $c_{1}\left(H^{-1}(E)\right)=n H$. Similarly, $H^{-1}(E)$ and $H^{0}(E) / \operatorname{tors}\left(H^{0}(E)\right)$ are $H$-stable, and if $H^{-1}(E)$ were not locally free, then (as in Lemma 3.2) there would be a subobject

$$
H^{-1}(E)^{* *} / H^{-1}(E) \subset H^{-1}(E)[1] \subset E
$$

of infinite slope, contradicting $t$-stability of $E$ for each value of $t$. This gives (2) and (3).

Finally, let $E^{\prime}$ be the kernel of the short exact sequence

$$
0 \rightarrow E^{\prime} \rightarrow E \rightarrow H^{0}(E) / \operatorname{tors}\left(H^{0}(E)\right) \rightarrow 0
$$

in $\mathcal{A}^{\#}$, so $H^{-1}\left(E^{\prime}\right)=H^{-1}(E)$ is locally free, and $H^{0}\left(E^{\prime}\right)=\operatorname{tors}\left(H^{0}(E)\right)$ is supported in dimension 0 . Then (4) follows directly from

Lemma 3.4. Suppose $E$ is an object of $\mathcal{A}^{\#}$ such that $H^{-1}(E)$ is locally free, and $H^{0}(E)$ is torsion, supported in dimension 0. Then either $E^{\vee}[1]$ is a torsion-free sheaf, or $E$ has a torsion subsheaf supported in dimension 0. 
Proof. Basically, this consists of taking duals twice. First, the dual of the sequence

$$
0 \rightarrow H^{-1}(E)[1] \rightarrow E \rightarrow H^{0}(E) \rightarrow 0
$$

in $\mathcal{A}^{\#}$ is a distinguished triangle

$$
\cdots \rightarrow H^{0}(E)^{\vee} \rightarrow E^{\vee} \rightarrow H^{-1}(E)^{\vee}[-1] \rightarrow H^{0}(E)^{\vee}[1] \rightarrow \cdots,
$$

whose associated sequence of cohomology sheaves is

$$
0 \rightarrow H^{1}\left(E^{\vee}\right) \rightarrow H^{-1}(E)^{*} \rightarrow H^{2}\left(H^{0}(E)^{\vee}\right) \rightarrow H^{2}\left(E^{\vee}\right) \rightarrow 0
$$

because of the assumptions on $H^{-1}(E)$ and $H^{0}(E)$. Thus either $H^{2}\left(E^{\vee}\right)=0$ and $E^{\vee}[1]=H^{1}\left(E^{\vee}\right)$ is a subsheaf of the dual vector bundle $H^{-1}(E)^{*}$, or else $H^{2}\left(E^{\vee}\right) \neq 0$ is the quotient of a sheaf supported in dimension 0 , hence is itself a sheaf supported in dimension 0. But in the latter case, the distinguished triangle

$$
\cdots \rightarrow H^{1}\left(E^{\vee}\right)[-1] \rightarrow E^{\vee} \rightarrow H^{2}\left(E^{\vee}\right)[-2] \rightarrow H^{1}\left(E^{\vee}\right) \rightarrow \cdots
$$

(coming from the fact that $E^{\vee}$ has cohomology only in degrees 1 and 2) dualizes to

$$
\cdots \rightarrow H^{2}\left(E^{\vee}\right)^{\vee}[2] \rightarrow E \rightarrow H^{1}\left(E^{\vee}\right)^{\vee}[1] \rightarrow \cdots,
$$

which is an exact sequence in $\mathcal{A}^{\#}$ ! And if $H^{2}\left(E^{\vee}\right) \neq 0$, then $H^{2}\left(H^{2}\left(E^{\vee}\right)^{\vee}\right) \neq 0$ as well.

Remark. Figuring out which of the objects of Proposition 3.3 are $t$-stable for each particular $t$ is, of course, more delicate. For example:

Lemma 3.5. A sheaf $i_{*} L_{C}$ as in Proposition 3.3 is $t$-stable unless there is an $H$-stable torsion-free sheaf $K$ on $S$ of rank $2 n+1$ with $c_{1}(K)=(n+1) H, \operatorname{Re}\left(Z_{t}(K)\right) \leq 0$ and a map $f: K \rightarrow i_{*} L_{C}$ that is generically (on $C$ ) surjective with a locally free kernel.

Proof. On the one hand, such a sheaf (and map) does destabilize $i_{*} L_{C}$, since the sequence $0 \rightarrow \operatorname{ker}(f) \rightarrow K \rightarrow i_{*} L_{C} \rightarrow \mathcal{O}_{Z} \rightarrow 0$ is a short exact sequence of objects in $\mathcal{A}^{\#}$,

$$
0 \rightarrow K \rightarrow i_{*} L_{C} \rightarrow Q \rightarrow 0
$$

(because $K \in \mathcal{T}$ and $F=\operatorname{ker}(f) \in \mathcal{F}$ ), where $Q \in \mathcal{A}$ satisfies $H^{-1}(Q)=\operatorname{ker}(f)$ and $H^{0}(Q)=\mathcal{O}_{Z}$. On the other hand, any potentially destabilizing sequence $0 \rightarrow K \rightarrow$ $i_{*} L_{C} \rightarrow Q \rightarrow 0$ gives rise to

$$
0 \rightarrow H^{-1}(Q) \rightarrow H^{0}(K) \rightarrow i_{*} L_{C} \rightarrow H^{0}(Q) \rightarrow 0 .
$$

From this we may read off:

- $H^{0}(Q)$ is supported in dimension 0 (otherwise $H^{-1}(Q)=0=H^{0}(K)$ ).

- $H^{0}(K)$ is torsion-free.

- $\operatorname{rk}\left(H^{-1}(Q)\right)=r=\operatorname{rk}\left(H^{0}(K)\right)$ and $c_{1}\left(H^{-1}(Q)\right)+H=c_{1}\left(H^{0}(K)\right)$. 
If $r=2 n$, then $c_{1}\left(H^{-1}(Q)\right)=n H$, and $Q$ has infinite slope. Thus, if the sequence is to destabilize $i_{*} L_{C}$, it must be the case that $\operatorname{rk}\left(H^{0}(K)\right)=2 n+1$ and $c_{1}\left(H^{0}(K)\right)=$ $(n+1) H$, and then it follows from $H^{0}(K) \in \mathcal{T}$ that $H^{0}(K)$ is $H$-stable. Finally, if $H^{-1}(Q)$ is not locally free, then $H^{-1}(Q)^{* *}$ has smaller slope, and $Q$ can be replaced by $Q^{\prime}$ with

$$
0 \rightarrow H^{-1}(Q)^{* *} / H^{-1}(Q) \rightarrow Q \rightarrow Q^{\prime} \rightarrow 0 \quad \text { and } \quad H^{-1}\left(Q^{\prime}\right)=H^{-1}(Q)^{* *} .
$$

Example. Let $L_{C}=\mathcal{O}_{C}\left(H+D-D^{\prime}\right)$ where $D, D^{\prime}$ are effective disjoint divisors of the same degree supported on the smooth part of $C$. Then

$$
\left.0 \rightarrow \mathcal{O}_{S} \rightarrow \mathcal{J}_{D^{\prime}}(H) \rightarrow i_{*} L_{C} \rightarrow L_{C}\right|_{D} \rightarrow 0
$$

(thinking of $D, D^{\prime}$ as zero-dimensional subschemes of $S$ ) will $t$-destabilize $i_{*} L_{C}$ if

$$
\operatorname{Re}\left(Z_{t}\left(\mathcal{J}_{D^{\prime}}(H)\right)\right)=\operatorname{deg}\left(D^{\prime}\right)+t^{2} H^{2} / 2-H^{2} / 8 \leq 0
$$

or in other words, if

$$
t^{2} \leq 1 / 4-\operatorname{deg}\left(D^{\prime}\right) \cdot 2 / H^{2},
$$

and if equality holds, then $i_{*} L_{C}$ will be an extension of stable objects of $\mathcal{A}^{\#}$ of the same phase (i.e. $i_{*} L_{C}$ is $t$-semistable, and $t$ is a critical value).

One final lemma on $t$-stability will be useful for us:

Lemma 3.6. Suppose $E$ is an object of $\mathcal{A}^{\#}$ which is an extension (in $\mathcal{A}^{\#}$ ) of the form

$$
0 \rightarrow \mathcal{J}_{W}^{\vee}[1] \rightarrow E \rightarrow \mathcal{J}_{Z}(H) \rightarrow 0
$$

for zero-dimensional schemes $Z, W$ with $\operatorname{len}(W)=\operatorname{len}(Z)$ (the rank-one case of Proposition 3.3). Then $E$ is $t$-stable unless either $\operatorname{Re}\left(Z_{t}\left(\mathcal{J}_{Z}(H)\right)\right) \geq 0$ and the quotient $E \rightarrow$ $\mathcal{J}_{Z}(H)$ destabilizes $E$, or else there is a sheaf $K \subset E$ as in Lemma 3.5.

Proof. A potentially $t$-destabilizing sequence $0 \rightarrow K \rightarrow E \rightarrow Q \rightarrow 0$ gives rise to

$$
0 \rightarrow H^{-1}(K) \rightarrow \mathcal{O}_{S} \rightarrow H^{-1}(Q) \rightarrow H^{0}(K) \rightarrow H^{0}(E) \rightarrow H^{0}(Q) \rightarrow 0 .
$$

The (by now) standard analysis allows us to conclude that if the sequence actually destabilizes $E$, then either

- $H^{-1}(K)=0, H^{0}(Q)$ is torsion supported in dimension 0 , and $H^{0}(K)=K$ of the lemma, or

- $H^{-1}(K)=0, H^{0}(Q)=\mathcal{J}_{Z}(H)$, and $\operatorname{rk}\left(H^{-1}(Q)\right)=2 n, c_{1}\left(H^{-1}(Q)\right)=n H$.

But in the latter case, the slope of $H^{0}(Q)=\mathcal{J}_{Z}(H)$ is smaller than the slope of $Q$, so if $Q$ destabilizes $E$, then so does $\mathcal{J}_{Z}(H)$ (only more so!).

Theorem 3.7. The objects $E$ of $\mathcal{A}^{\#}$ with numerical invariants

$$
\operatorname{ch}_{0}(E)=0, \quad \operatorname{ch}_{1}(E)=H, \quad \operatorname{ch}_{2}(E)=H^{2} / 2
$$

that are $t$-stable for some $t>1 / 6$ are either 
- sheaves of the form $i_{*} L_{C}$ (as in Proposition 3.3), or else

- fit into (non-split!) extensions of the form

$$
0 \rightarrow \mathcal{J}_{W}^{\vee}[1] \rightarrow E \rightarrow \mathcal{J}_{Z}(H) \rightarrow 0
$$

where $Z, W \subset S$ are zero-dimensional subschemes of the same length.

Moreover, if $E$ is one of the objects above, and $E$ is not $t$-stable for some $t>1 / 6$, then $E$ is destabilized by a twisted ideal sheaf $\mathcal{J}_{Y}(H) \subset E$ for some zero-dimensional subscheme $Y \subset S$.

Proof. By Proposition 3.3, any $t$-stable object is either of the form $i_{*} L_{C}$ or else fits in a sequence $0 \rightarrow K \rightarrow E \rightarrow Q \rightarrow 0$ where $Q$ is an $H$-stable torsion-free sheaf of odd rank $2 n+1$ and $c_{1}=(n+1) H$ and $K$ is the shifted derived dual of a torsion-free sheaf. But by the Bogomolov-Gieseker inequality,

$$
\operatorname{ch}_{2}(Q) \leq \frac{(n+1)^{2} H^{2}}{2(2 n+1)},
$$

and then

$$
\operatorname{Re}\left(Z_{t}(Q)\right) \geq \frac{H^{2}}{2}\left(t^{2}(2 n+1)-\frac{1}{4(2 n+1)}\right) .
$$

Thus if $t \geq 1 / 2$, there are no such $t$-stable objects (so the only $t$-stable objects are of the form $i_{*} L_{C}$ ), if $t \geq 1 / 6$, there are none such with $r=2 n+1 \geq 3$, etc.

The last part of the theorem now follows from Lemmas 3.5 and 3.6.

\section{Families and walls}

For $t>1 / 2$, the moduli of $t$-stable objects of $\mathcal{A}^{\#}$ is the moduli space

$$
\mathcal{M}:=\mathcal{M}_{S}\left(0, H, H^{2} / 2\right)
$$

of (Gieseker-stable) coherent sheaves on $S$ of the form $i_{*} L_{C}$. As $t$ crosses the critical values

$$
1 / 2, \sqrt{1 / 4-2 / H^{2}}, \sqrt{1 / 4-2 \cdot 2 / H^{2}}, \ldots>1 / 6,
$$

the $t$-stability changes, as subobjects of certain coherent sheaves $i_{*} L_{C}$ (or more generally, objects of $\mathcal{A}^{\#}$ from Lemma 3.6) of the form $\mathcal{J}_{Z}(H)$ achieve zero (and then positive) slope. The resulting birational modifications of $\mathcal{M}$ as $t$ passes over critical points can be predicted, but are only carried out (in §5) in case $S$ is $K$-trivial, because it is only in that case that we can prove that the desired birational transformations (which are then Mukai flops) actually exist.

Definition. For (quasi-projective) schemes $X$, the objects $\varepsilon_{X}$ of the bounded derived category $\mathcal{D}(S \times X)$ are families of objects of $\mathcal{D}(S)$ parametrized by $X$. 
Definition. A family $\mathcal{E}_{X}$ is a flat family of objects of $\mathcal{A}^{\#}$ if the (derived) restrictions to the fibers

$$
\mathcal{E}_{x}:=L i_{S \times x}^{*} \mathcal{E}
$$

are objects of $\mathcal{A}^{\#}$ for all closed points $x \in X$ (via the isomorphism $S \times x \cong S$ ).

Remark. A flat family of coherent sheaves on $S$ is a coherent sheaf on $S \times X$ that restricts to a coherent sheaf (i.e. there are no higher tors) on each $S \times x$. Thus, this is a natural analogue. As in the case of flat families of coherent sheaves, these objects do not form an abelian category (cokernels of morphisms of flat families need not be flat families), but Abramovich-Polishchuk define an analogue of the full abelian category of coherent sheaves on $X \times S$ [AP06] (at least in the case where $X$ is smooth). We will not need to make use of this.

Example. The universal family of coherent sheaves $\mathcal{U} \rightarrow S \times \mathcal{M}$ for the moduli space $\mathcal{M}=\mathcal{M}_{S}\left(0, H, H^{2} / 2\right)$ is a flat family of objects of $\mathcal{A}^{\#}$ (all coherent sheaves!).

Example. Let $S[d]$ be the Hilbert scheme of length $d$ subschemes of $S$, with universal subscheme $\mathcal{Z} \subset S \times S[d]$. Then the sheaf $\mathcal{J}_{\mathcal{Z}}(H):=\mathcal{J}_{\mathcal{Z}} \otimes \pi_{1}^{*} \mathcal{O}_{S}(H)$ is a flat family of objects of $\mathcal{A}^{\#}$ (and of coherent sheaves).

Example. The shifted derived dual $\mathcal{J}_{Z}^{\vee}[1]$ (in $\mathcal{D}(S \times S[d])$ ) is a flat family of objects of $\mathcal{A}^{\#}$.

Indeed, it is a consequence of the flatness of the coherent sheaf $\mathcal{J}_{\mathcal{Z}}$ over $S[d]$ that $L i_{S \times\{Z\}}^{*} \mathcal{J}_{Z}^{\vee}[1]=\mathcal{J}_{Z}^{\vee}[1]$ for each $Z \in S[d]$.

Our goal is to produce flat families of objects of $\mathcal{A}^{\#}$ parametrizing extensions of the form

$$
0 \rightarrow \mathcal{J}_{Z}(H) \rightarrow E \rightarrow \mathcal{J}_{W}^{\vee}[1] \rightarrow 0 \quad \text { and } \quad 0 \rightarrow \mathcal{J}_{W}^{\vee}[1] \rightarrow E \rightarrow \mathcal{J}_{Z}(H) \rightarrow 0
$$

that are exchanged under the wall-crossing. These will both be projective bundles when $S$ is $K$-trivial, thanks to the following vanishing result:

Proposition 4.1. Let $S$ be a smooth surface with $\operatorname{Pic}(S)=\mathbb{Z}[H]$. Then

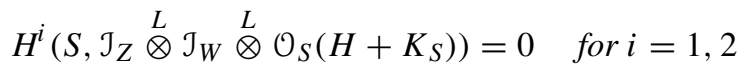

for all subschemes $Z, W \subset S$ of (the same) length d provided that

$$
d<H^{2} / 8 \text {. }
$$

Remark. In the case $d=1$, this is a weak form of Reider's Theorem [Fri98], since it amounts to saying that $H+K_{S}$ is very ample if $H$ is ample, generating $\operatorname{Pic}(S)$, and $H^{2} \geq 9$. 
Proof. In the derived category $\mathcal{D}(S)$,

$$
H^{i}\left(S, \mathcal{J}_{Z} \stackrel{L}{\otimes} \mathcal{J}_{W} \stackrel{L}{\otimes} \mathcal{O}_{S}\left(H+K_{S}\right)\right) \cong \operatorname{Ext}_{\mathcal{D}(S)}^{i+1}\left(\mathcal{J}_{W}^{\vee}[1], \mathcal{J}_{Z}\left(H+K_{S}\right)\right),
$$

and by Grothendieck duality,

$$
\operatorname{Ext}_{\mathcal{D}(S)}^{i+1}\left(\mathcal{J}_{W}^{\vee}[1], \mathcal{J}_{Z}\left(H+K_{S}\right)\right) \cong \operatorname{Ext}_{\mathcal{A}^{\#}}^{1-i}\left(\mathcal{J}_{Z}(H), \mathcal{J}_{W}^{\vee}[1]\right)^{*}
$$

This immediately gives $H^{2}\left(S, \mathcal{J}_{Z} \otimes \mathcal{J}_{W} \otimes \mathcal{O}_{S}\left(H+K_{S}\right)\right)=0$ (which was easy to check anyway), and identifies the space of extensions of $\mathcal{J}_{Z}(H)$ by $\mathcal{J}_{W}^{\vee}[1]$ with $H^{0}\left(S, \mathcal{J}_{Z} \otimes \mathcal{J}_{W} \otimes\right.$ $\left.\mathcal{O}_{S}\left(H+K_{S}\right)\right)^{*}$. But it also identifies

$$
H^{1}\left(S, \mathcal{J}_{Z} \otimes \mathcal{J}_{W} \otimes \mathcal{O}_{S}\left(H+K_{S}\right)\right)^{*} \cong \operatorname{Hom}_{\mathcal{A}^{\#}}\left(\mathcal{J}_{Z}(H), \mathcal{J}_{W}^{\vee}[1]\right),
$$

and we may conclude that this is zero if we can find a value of $t>0$ such that

$$
\mathcal{J}_{Z}(H), \mathcal{J}_{W}^{\vee}[1] \text { are both } t \text {-stable and } \mu_{t}\left(\mathcal{J}_{Z}(H)\right)>\mu_{t}\left(\mathcal{J}_{W}^{\vee}[1]\right) \text {. }
$$

But the "wall" where these two slopes coincide is precisely at $t=\sqrt{1 / 4-d \cdot 2 / H^{2}}$. which satisfies $t>0$ when $d<H^{2} / 8$, as desired.

Remark. It is interesting that this vanishing theorem can be proved using stability conditions, following more or less immediately from the Hodge Index Theorem and the Bogomolov-Gieseker inequality. A stronger inequality valid for K3 surfaces will give a stronger vanishing result in $§ 6$.

Proposition 4.2. Assume the vanishing of Proposition 4.1. If $K_{S} \geq 0$, the projective bundle

$$
\mathbb{P}_{d} \rightarrow S[d] \times S[d] \quad \text { with fibers } \mathbb{P}\left(H^{0}\left(S, \mathcal{J}_{Z} \otimes \mathcal{J}_{W}\left(H+K_{S}\right)\right)\right)
$$

supports a universal family $\mathcal{E}_{d}$ (on $S \times \mathbb{P}_{d}$ ) of extensions of objects of $\mathcal{A}^{\#}$ of the form

$$
0 \rightarrow \mathcal{J}_{Z}\left(H+K_{S}\right) \rightarrow E \rightarrow \mathcal{J}_{W}^{\vee}[1] \rightarrow 0 .
$$

For any $S$, the dual projective bundle $\mathbb{P}_{d}^{\vee}$ supports a universal family $\mathcal{F}_{d}$ of extensions of the form

$$
0 \rightarrow \mathcal{J}_{W}^{\vee}[1] \rightarrow F \rightarrow \mathcal{J}_{Z}(H) \rightarrow 0
$$

Proof. Let

$$
z_{12}, z_{13} \subset S \times S[d] \times S[d]
$$

be the pull-backs of $z \subset S \times S[d]$ via the projections $\pi_{12}, \pi_{13}: S \times S[d] \times S[d] \rightarrow$ $S \times S[d]$ and consider the (a priori derived) object

$$
\operatorname{RHom}\left(\mathcal{J}_{Z_{13}}^{\vee}[1], \mathcal{J}_{Z_{12}}\left(H+K_{S}\right)\right)[1] \cong \mathcal{J}_{Z_{13}} \stackrel{L}{\otimes} \mathcal{J}_{Z_{12}}\left(H+K_{S}\right) .
$$

Since the ideal sheaf $\mathcal{J}_{Z}$ admits a two-step resolution by vector bundles (see [ES98]) it follows that $\mathcal{J}_{z_{13}} \stackrel{L}{\otimes} \mathcal{J}_{z_{12}}(H)$ is (equivalent to) a flat coherent sheaf over $S[d] \times S[d]$. 
Let $\pi: S \times S[d] \times S[d] \rightarrow S[d] \times S[d]$ be the projection. We may set

$$
\mathbb{P}_{d}:=\mathbb{P}\left(\pi_{*}\left(\mathcal{J}_{Z_{13}} \otimes \mathcal{J}_{Z_{12}}\left(H+K_{S}\right)\right)\right)
$$

since the push-forward is locally free, by base change.

Next, we turn to the construction of the universal family on $S \times \mathbb{P}_{d}$. This should morally be thought of as a single extension of the form

$$
0 \rightarrow \rho^{*} \mathcal{J}_{Z_{12}}\left(H+K_{S}\right) \rightarrow \mathcal{E}_{d} \rightarrow \rho^{*} \mathcal{J}_{Z_{13}}^{\vee}[1] \otimes \mathcal{O}_{\mathbb{P}_{d}}(-1) \rightarrow 0
$$

where

$$
\rho: S \times \mathbb{P}_{d} \rightarrow S \times S[d] \times S[d]
$$

is the projection. But we have avoided any mention of an abelian category of objects on $S \times \mathbb{P}_{d}$ containing both $\rho^{*} \mathcal{J}_{Z_{12}}\left(H+K_{S}\right)$ and $\rho^{*} \mathcal{J}_{Z_{13}}^{\vee}[1] \otimes \mathcal{O}_{\mathbb{P}_{d}}(-1)$. Instead, we make the construction using distinguished triangles. There is a canonical element

$$
\begin{aligned}
\mathrm{id} & \in \Gamma\left(S \times \mathbb{P}_{d}, \rho^{*}\left(\mathcal{J}_{Z_{13}} \otimes \mathcal{J}_{Z_{12}}\left(H+K_{S}\right)\right) \otimes \mathcal{O}_{\mathbb{P}_{d}}(1)\right) \\
& =\Gamma\left(S \times S[d] \times S[d], \mathcal{J}_{Z_{13}} \otimes \mathcal{J}_{Z_{12}}\left(H+K_{S}\right) \otimes \rho_{*} \mathcal{O}_{\mathbb{P}_{d}}(1)\right) \\
& =\Gamma\left(S[d] \times S[d], \pi_{*}\left(\mathcal{J}_{Z_{13}} \otimes \mathcal{J}_{Z_{12}}\left(H+K_{S}\right)\right) \otimes \pi_{*}\left(\mathcal{J}_{Z_{13}} \otimes \mathcal{J}_{Z_{12}}\left(H+K_{S}\right)\right)^{*}\right),
\end{aligned}
$$

which can be alternatively thought of as the canonical element

$$
f_{\text {id }} \in \operatorname{RHom}_{S \times \mathbb{P}_{d}}\left(\rho^{*} \mathcal{J}_{\mathcal{Z}_{13}}^{\vee} \otimes \mathcal{O}_{\mathbb{P}_{d}}(-1), \rho^{*} \mathcal{J}_{Z_{12}}\left(H+K_{S}\right)\right) .
$$

With this canonical element, we form the cone and distinguished triangle

$$
\begin{aligned}
\cdots \rightarrow \rho^{*} \mathcal{J}_{Z_{13}}^{\vee} \otimes \mathcal{O}_{\mathbb{P}_{d}}(-1) \stackrel{f_{\mathrm{id}}}{\longrightarrow} \rho^{*} \mathcal{J}_{Z_{12}} & \left(H+K_{S}\right) \rightarrow \mathcal{E}_{d} \\
& \rightarrow \rho^{*} \mathcal{J}_{Z_{13}}^{\vee}[1] \otimes \mathcal{O}_{\mathbb{P}_{d}}(-1) \stackrel{f_{\text {id }}[1]}{\longrightarrow} \cdots .
\end{aligned}
$$

If $K_{S} \geq 0$, then each $\mathcal{J}_{Z}\left(H+K_{S}\right)$ is an object of $\mathcal{A}^{\#}=\mathcal{A}_{\left(\frac{1}{2} H, t H\right)}^{\#}$ and then this "universal" distinguished triangle has the property that each $L i_{S \times \epsilon}^{*}(* *)$,

$$
\left.\cdots \rightarrow \mathcal{J}_{Z}\left(H+K_{S}\right) \rightarrow \mathcal{E}_{d}\right|_{S \times \epsilon} \rightarrow \mathcal{J}_{W}^{\vee}[1] \rightarrow \cdots,
$$

is the short exact sequence (in $\mathcal{A}^{\#}$ ) corresponding to the extension

$$
\epsilon \in \mathbb{P}\left(H^{0}\left(S, \mathcal{J}_{W} \otimes \mathcal{J}_{Z}\left(H+K_{S}\right)\right)\right) \cong \mathbb{P}\left(\operatorname{Ext}_{\mathcal{A}^{\#}}^{1}\left(\mathcal{J}_{W}^{\vee}[1], \mathcal{J}_{Z}\left(H+K_{S}\right)\right)\right)
$$

Turning next to the family $\mathcal{F}_{d}$, we define, similarly,

$$
\rho^{\vee}: S \times \mathbb{P}_{d}^{\vee} \rightarrow S \times S[d] \times S[d],
$$

and as above, the key point is the existence of a canonical morphism

$$
f_{\text {id }}^{\vee}:\left(\rho^{\vee}\right)^{*} \mathcal{J}_{Z_{12}}(H)[-1] \rightarrow\left(\rho^{\vee}\right)^{*} \mathcal{J}_{Z_{13}}^{\vee}[1] \otimes \mathcal{O}_{\mathbb{P}_{d}^{\vee}}(1),
$$


which will, in turn, define the distinguished triangle

$\cdots \rightarrow\left(\rho^{\vee}\right)^{*} \mathcal{J}_{\mathcal{Z}_{12}}(H)[-1] \rightarrow\left(\rho^{\vee}\right)^{*} \mathcal{J}_{\mathcal{Z}_{13}}^{\vee}[1] \otimes \mathcal{O}_{\mathbb{P}_{d}^{\vee}}(1) \rightarrow \mathcal{F}_{d} \rightarrow\left(\rho^{\vee}\right)^{*} \mathcal{J}_{\mathcal{Z}_{12}}(H) \rightarrow \cdots$,

which is the desired universal family (whether or not $K_{S} \geq 0$ !).

But this canonical morphism is obtained from Serre duality [Cal05]:

$$
\begin{aligned}
& \operatorname{RHom}\left(\left(\rho^{\vee}\right)^{*} \mathcal{J}_{\mathcal{Z}_{12}}(H)[-1],\left(\rho^{\vee}\right)^{*} \mathcal{J}_{\mathcal{Z}_{13}}^{\vee}[1] \otimes \mathcal{O}_{\mathbb{P}_{d}^{\vee}}(1)\right) \\
& \cong \operatorname{RHom}\left(\left(\rho^{\vee}\right)^{*}\left(\mathcal{J}_{\mathcal{Z}_{12}} \otimes \mathcal{J}_{\mathcal{Z}_{13}}(H)\right), \mathcal{O}_{\mathbb{P}_{d}^{\vee}}(1)[2]\right) \\
& \cong \operatorname{RHom}\left(\left(\rho^{\vee}\right)^{*}\left(\mathcal{J}_{\mathcal{Z}_{12}} \otimes \mathcal{J}_{\mathcal{Z}_{13}}\left(H+K_{S}\right)\right), \mathcal{O}_{\mathbb{P}_{d}^{\vee}}(1) \otimes \mathcal{O}_{S}\left(K_{S}\right)[2]\right) \\
&=\operatorname{RHom}\left(\left(\rho^{\vee}\right)^{*}\left(\mathcal{J}_{\mathcal{Z}_{12}} \otimes \mathcal{J}_{\mathcal{Z}_{13}}\left(H+K_{S}\right)\right), \pi^{!} \mathcal{O}_{\mathbb{P}_{d}^{\vee}}(1)\right) \\
& \cong \operatorname{RHom}\left(\pi_{*}\left(\mathcal{J}_{\mathcal{Z}_{12}} \otimes \mathcal{J}_{\mathcal{Z}_{13}}\left(H+K_{S}\right)\right), \pi_{*}\left(\mathcal{J}_{\mathcal{Z}_{12}} \otimes \mathcal{J}_{\mathcal{Z}_{13}}\left(H+K_{S}\right)\right)\right) .
\end{aligned}
$$

\section{Mukai flops}

In this section, and for the rest of the paper, we assume that $K_{S}=0$, for the following reason.

Moduli spaces $\mathcal{M}=\mathcal{M}_{S}\left(r, c_{1}, \mathrm{ch}_{2}\right)$ of $H$-stable coherent sheaves on a $K$-trivial surface are symplectic, meaning that there is a skew-symmetric isomorphism on the tangent bundle

$$
\omega: T \mathcal{M} \rightarrow T^{*} \mathcal{M} \text {. }
$$

The form is given by the natural isomorphism of Serre duality (see [Muk84])

$$
\operatorname{Ext}_{\mathcal{O}_{S}}^{1}(E, E) \cong \operatorname{Ext}_{\mathcal{O}_{S}}^{1}(E, E)^{*}
$$

In fact, this argument applies as well to moduli spaces of stable objects of $\mathcal{A}^{\#}$, taking $\operatorname{Ext}_{\mathcal{A}^{\#}}^{1}(E, E)$, once such moduli spaces are shown to exist (see [In10]).

Varieties with a symplectic structure are necessarily even-dimensional. When such a variety is equipped with an appropriate "Lagrangian" subvariety, then it always admits an elementary birational transformation (Mukai flop):

Theorem (Theorem 0.7 of [Muk84]). Let $X$ be a symplectic variety, and let $P$ be a $\mathbb{P}^{n}$ bundle, over a base $B$, contained in $X$ in codimension $n \geq 2$. Then there is a birational map, denoted $\operatorname{elm}_{P}: X \rightarrow X^{\prime}$, with the following properties:

(1) $X^{\prime}$ contains the dual $\mathbb{P}^{n}$ bundle $P^{\prime}$ over $B$ and has a symplectic structure $\omega^{\prime}$ which coincides with $\omega$ outside of $P^{\prime}$.

(2) $\operatorname{elm}_{P}$ is the composite of the blowing up $\sigma^{-1}: X \rightarrow \tilde{X}$ along $P$ and the blowing down $\sigma^{\prime}: \widetilde{X} \rightarrow X^{\prime}$ of the exceptional divisor $D=\sigma^{-1}(P)$ onto $P^{\prime}$. Moreover, $D \subset$ $P \times_{B} P^{\prime}$ is the two-step flag bundle over $B$, and $\left.\left.\mathcal{O}_{\widetilde{X}}(D)\right|_{D} \cong \mathcal{O}_{P \times_{B} P^{\prime}}(-1,-1)\right|_{D}$. 
Theorem 5.1. Fix $S$ with $K_{S}=0$ and $\operatorname{Pic}(S)=\mathbb{Z}[H]$ (i.e. $S$ is a $K 3$ surface) and let

$$
\left\{t_{d}=\sqrt{1 / 4-d \cdot 2 / H^{2}} \mid d=0,1,2, \ldots<H^{2} / 8\right\}
$$

be the set of "rank one" critical values for stability conditions $\left(Z_{t}, \mathcal{A}^{\#}\right)$. Then for each $t>1 / 6$ and away from the critical set, there is a smooth, proper moduli space

$$
\mathcal{M}_{t}:=\mathcal{M}_{t}\left(0, H, H^{2} / 2\right)
$$

and a coherent sheaf $\mathcal{U}_{t}$ on $S \times \mathcal{M}_{t}$ which together represent the functor

$$
X \mapsto\left\{\text { equivalence classes of flat families of t-stable objects of } \mathcal{A}^{\#}\right. \text { over X\} }
$$

where two such flat families $\mathcal{E}_{X}, \mathcal{E}_{X}^{\prime} \in D^{b}(S \times X)$ are equivalent (as in the case of coherent sheaves) if there is a line bundle $\mathcal{L}$ on $X$ and an isomorphism $\mathcal{E}_{X} \cong \mathcal{E}_{X}^{\prime} \otimes \pi_{X}^{*} \mathcal{L}$ in $D^{b}(S \times X)$.

Proof. The moduli space $\mathcal{M}_{t}$ for $t>1 / 2$ is the "classical" space $\mathcal{M}_{S}\left(0, H, H^{2} / 2\right)$ of rank one torsion-free sheaves of degree $H^{2}$ on curves $C \in|H|$. This admits a universal coherent sheaf by geometric invariant theory. The general proof consists of three parts, which carry out an induction that constructs each $\mathcal{M}_{t_{d}-\epsilon}$ out of $\mathcal{M}_{t_{d}+\epsilon}\left(=\mathcal{M}_{t_{d-1}-\epsilon}\right)$ near each of the "walls" $t_{d}>1 / 6$.

Assume that $t_{d}>1 / 6$ and $\mathcal{M}_{t_{d}+\epsilon}$, together with "universal" coherent sheaf $\mathcal{U}_{t_{d}+\epsilon}$ on $S \times \mathcal{M}_{t_{d}+\epsilon}$, is the smooth, proper moduli space representing the functor of isomorphism classes of $t_{d}+\epsilon$-stable objects with the given invariants. Then:

Step 1: There is a natural embedding $\mathbb{P}_{d} \subset \mathcal{M}_{t_{d}+\epsilon}$ of the projective bundle from Theorem 4.2 that parametrizes all the objects of $\mathcal{M}_{t_{d}+\epsilon}$ that are not $t_{d}-\epsilon$-stable.

Interlude: Construct $\mathbb{P}_{d}^{\vee} \subset \mathcal{M}^{\prime}$ as the Mukai flop of $\mathbb{P}_{d} \subset \mathcal{M}:=\mathcal{M}_{t_{d}+\epsilon}$.

Step 2: There is a coherent sheaf $\mathfrak{U}^{\prime}$ on $S \times \mathcal{M}^{\prime}$ naturally obtained as the "Radon transform" across the Mukai flop of the universal coherent sheaf $\mathcal{U}_{t_{d}+\epsilon}$ on $S \times \mathcal{M}_{t_{d}+\epsilon}$, such that:

Step 3: $\mathcal{M}^{\prime}$ together with the sheaf $\mathcal{U}^{\prime}$ is the desired $\mathcal{M}_{t_{d}-\epsilon}$ (and family $\mathcal{U}_{t_{d}-\epsilon}$ ).

Proof of Step 1. If $Z, W \subset S$ are subschemes of length $d$, consider an object $E$ of $\mathcal{A}^{\#}$ given as an extension

$$
0 \rightarrow \mathcal{J}_{Z}(H) \rightarrow E \rightarrow \mathcal{J}_{W}^{\vee}[1] \rightarrow 0 .
$$

First, recall that both $\mathcal{J}_{Z}(H)$ and $\mathcal{J}_{W}^{\vee}[1]$ are $t$-stable (Lemma 3.1). Since

$$
\operatorname{Re}\left(Z_{t}\left(\mathcal{J}_{Z}(H)\right)\right)=d+\left(H^{2} / 2\right)\left(t^{2}-1 / 4\right) \quad \text { and } \quad \operatorname{Re}\left(Z_{t}\left(\mathcal{J}_{W}^{\vee}[1]\right)\right)=-\operatorname{Re}\left(Z_{t}\left(\mathcal{J}_{Z}(H)\right)\right)
$$

it follows that $E$ is not $t$-stable if $t \leq t_{d}$ (recall that $t_{d}$ solves $\left.d+\left(H^{2} / 2\right)\left(t_{d}^{2}-1 / 4\right)=0\right)$. But we claim that $E$ is $t$-stable for $t_{d}<t<t_{d-1}$. To see this, consider the cohomology sequence of sheaves associated to the extension defining $E$,

$$
0 \rightarrow H^{-1}(E) \rightarrow \mathcal{O}_{S} \rightarrow \mathcal{J}_{Z}(H) \rightarrow H^{0}(E) \rightarrow T \rightarrow 0
$$


From this it follows that either $H^{-1}(E)=0$, in which case $E=H^{0}(E)=i_{*} L_{C}$ for some (necessarily torsion-free!) rank one sheaf on $C$, or else $H^{-1}(E)=\mathcal{O}_{S}$, and then $E$ fits in an extension

$$
0 \rightarrow \mathcal{J}_{W^{\prime}}^{\vee}[1] \rightarrow E \rightarrow \mathcal{J}_{Z^{\prime}}(H) \rightarrow 0
$$

where $\mathcal{J}_{Z^{\prime}}(H)=H^{0}(E) / \operatorname{tors}\left(H^{0}(E)\right)$. Moreover, this sequence only splits if $Z=Z^{\prime}$ and $W=W^{\prime}$, and the original exact sequence is split. Thus the second part of Theorem 3.7 applies, and if $E$ were not $t$ stable, then it would be destabilized by an ideal sheaf $\mathcal{J}_{Y}(H) \subset E$. On the other hand, such ideal sheaves satisfy $\operatorname{Re}\left(Z_{t}\left(\mathcal{J}_{Y}(H)\right)\right)=$ $d^{\prime}+\left(H^{2} / 2\right)\left(t^{2}-1 / 4\right)$, where $d^{\prime}=\operatorname{len}(Y)$, so if $E$ were destabilized by such a sheaf, and if $t_{d}<t<t_{d-1}$, then len $(Y) \leq d-1$, the induced map $\mathcal{J}_{Y}(H) \rightarrow \mathcal{J}_{W}^{\vee}[1]$ is the zero map (otherwise it would destabilize $\mathcal{J}_{W}^{\vee}[1]$ !), and so $\mathcal{J}_{Y}(H) \subset \mathcal{J}_{Z}(H)$, which contradicts the fact that len $(Y)<\operatorname{len}(Z)$.

Thus the non-split extensions parametrized by $\mathbb{P}_{d}$ produce $t$-stable objects (for $t_{d}<$ $\left.t<t_{d-1}\right)$ and the family of Theorem 4.2 defines a morphism $i_{d}: \mathbb{P}_{d} \rightarrow \mathcal{M}_{t}$ We claim that $i_{d}$ is an embedding. First, if $i_{d}(\epsilon)=i_{d}\left(\epsilon^{\prime}\right)$, where $\epsilon, \epsilon^{\prime}$ are extension classes defining isomorphic objects $E, E^{\prime}$, then because $E$ and $E^{\prime}$ are both $t$-stable, it follows that the isomorphism is a multiple of the identity map. Moreover, since $\operatorname{Hom}\left(\mathcal{J}_{Z}(H), \mathcal{J}_{W^{\prime}}^{\vee}[1]\right)=0$ for all $Z, W$ satisfying len $(Z)=\operatorname{len}\left(W^{\prime}\right)=d$, it follows that the isomorphism $E \cong E^{\prime}$ induces vertical isomorphisms in the diagram

$$
\begin{aligned}
\epsilon: 0 & \rightarrow \mathcal{J}_{Z}(H) \rightarrow E \\
\|_{2} & \rightarrow \mathcal{J}_{W}^{\vee}[1] \rightarrow 0 \\
\epsilon^{\prime}: 0 & \rightarrow \mathcal{J}_{Z^{\prime}}(H) \rightarrow E^{\prime} \rightarrow \mathcal{J}_{W^{\prime}}^{\vee}[1] \rightarrow 0
\end{aligned}
$$

all of which are multiples of the identity. Thus $Z=Z^{\prime}, W=W^{\prime}$ and $\epsilon=\lambda \epsilon^{\prime}$ for some non-zero scalar $\lambda$. In other words, the equivalence classes of the extensions modulo scalars satisfy $[\epsilon]=\left[\epsilon^{\prime}\right]$ (in $\mathbb{P}_{d}$ ). Thus $i_{d}$ is injective.

To complete the proof that $i_{d}$ is an embedding, we need to study the induced map on tangent spaces. The tangent space to $\mathcal{M}_{t}$ at a point $E \in \mathcal{M}_{t}$ is easiest to describe. It is $\operatorname{Ext}_{\mathcal{A}^{\#}}^{1}(E, E)$ (the same as the tangent space to the stack; see Step 3). If $E=i_{d}([\epsilon])$, where

$$
\epsilon: 0 \rightarrow \mathcal{J}_{Z}(H) \rightarrow E \rightarrow \mathcal{J}_{W}^{\vee}[1] \rightarrow 0
$$

is a (non-split) extension, then $\operatorname{Ext}_{\mathcal{A}^{\#}}^{1}(E, E)$ fits into a long exact sequence

$$
0 \rightarrow V \rightarrow \operatorname{Ext}_{\mathcal{A}^{\#}}^{1}(E, E) \rightarrow \operatorname{Ext}_{\mathcal{A}^{\#}}^{1}\left(\mathcal{J}_{Z}(H), \mathcal{J}_{W}^{\vee}[1]\right) \stackrel{\epsilon^{\vee}}{\rightarrow} \operatorname{Ext}_{\mathcal{A}^{\#}}^{2}\left(\mathcal{J}_{W}^{\vee}[1], \mathcal{J}_{W}^{\vee}[1]\right)=\mathbb{C} \rightarrow 0
$$

where $V$ is identified with the tangent space to $\mathbb{P}_{d}$ at the point $[\epsilon]$ via

$$
\operatorname{Ext}_{\mathcal{A}^{\#}}^{1}\left(\mathcal{J}_{Z}(H), \mathcal{J}_{Z}(H)\right) \cong T_{S[d]}(Z), \quad \operatorname{Ext}_{\mathcal{A}^{\#}}^{1}\left(\mathcal{J}_{W}^{\vee}[1], \mathcal{J}_{W}^{\vee}[1]\right) \cong T_{S[d]}(W)
$$

and the canonical maps

$$
\begin{aligned}
0 \rightarrow \mathbb{C}=\operatorname{Hom}\left(\mathcal{J}_{Z}(H), \mathcal{J}_{Z}(H)\right) \stackrel{\epsilon}{\rightarrow} \operatorname{Ext}_{\mathcal{A}^{\#}}^{1}\left(\mathcal{J}_{W}^{\vee}[1], \mathcal{J}_{Z}(H)\right) \rightarrow V \\
\rightarrow \operatorname{Ext}_{\mathcal{A}^{\#}}^{1}\left(\mathcal{J}_{Z}(H), \mathcal{J}_{Z}(H)\right) \oplus \operatorname{Ext}_{\mathcal{A}^{\#}}^{1}\left(\mathcal{J}_{W}^{\vee}[1], \mathcal{J}_{W}^{\vee}[1]\right) \rightarrow 0
\end{aligned}
$$

induced from the extension class $\epsilon$. 
Thus, from the extension class itself, the inclusion of $V \cong T_{\mathbb{P}_{d}}([\epsilon])$ in $T_{\mathcal{M}}(E)$ is identified with the differential of $i_{d}$. In particular, $i_{d}$ is an embedding, and the normal space at $E$ is canonically identified with the kernel of the map

$$
\operatorname{Ext}_{\mathcal{A}^{\#}}^{1}\left(\mathcal{J}_{Z}(H), \mathcal{J}_{W}^{\vee}[1]\right) \cong \operatorname{Ext}^{1}\left(\mathcal{J}_{W}^{\vee}[1], \mathcal{J}_{Z}(H)\right)^{*} \stackrel{\epsilon^{\vee}}{\rightarrow} \mathbb{C} .
$$

Interlude. By induction (or else directly!), $\mathcal{M}_{t}$ is a symplectic variety, and

- $\operatorname{dim}\left(\mathcal{M}_{t}\right)=\operatorname{dim}\left(\operatorname{Ext}_{\mathcal{D}(S)}^{1}(E, E)\right)=2+H^{2}$,

- $\operatorname{dim}\left(\mathbb{P}_{d}\right)=2 \operatorname{dim}(S[d])+\left(\chi\left(S, \mathcal{J}_{Z} \otimes \mathcal{J}_{W} \otimes \mathcal{O}_{S}(H)\right)-1\right)=4 d+\left(1+H^{2} / 2-2 d\right)$,

so that, indeed, the embedding of Step 1 satisfies

$$
\operatorname{codim}\left(\mathbb{P}_{d}\right)=H^{2} / 2+1-2 d=\text { fiber dimension }
$$

and there is a Mukai flop $\mathbb{P}_{d}=P \subset \mathcal{M}:=\mathcal{M}_{t_{d}+\epsilon} \rightarrow \mathcal{M}^{\prime} \supset P^{\prime}$. We can now describe all the points of $\mathcal{M}^{\prime}$ :

- The points of $\mathcal{M}^{\prime}-P^{\prime}=\mathcal{M}-P$ correspond to all the objects of $\mathcal{A}^{\#}$ with the given invariants that are both $t_{d}+\epsilon$-stable and $t_{d}-\epsilon$-stable.

- Via the isomorphism $\operatorname{Ext}_{\mathcal{A}^{\#}}^{1}\left(\mathcal{J}_{Z}(H), \mathcal{J}_{W}^{\vee}[1]\right) \cong \operatorname{Ext}_{\mathcal{A}^{\#}}^{1}\left(\mathcal{J}_{W}^{\vee}[1], \mathcal{J}_{Z}(H)\right)^{*}$ the points of $P^{\prime}=\mathbb{P}_{d}^{\vee}$ correspond to non-zero extensions (modulo scalars) of the form

$$
0 \rightarrow \mathcal{J}_{W}^{\vee}[1] \rightarrow E \rightarrow \mathcal{J}_{Z}(H) \rightarrow 0
$$

with len $(W)=\operatorname{len}(Z)=d$.

It is evident that such extensions define objects $E$ of $\mathcal{A}^{\#}$ that are not $t_{d}+\epsilon$-stable. On the other hand, if $E$ is an object of $\mathcal{A}^{\#}$ with the given invariants that is $t_{d}-\epsilon$-stable, then by Theorem 3.7, $E$ is either of the form $i_{*} L_{C}$ or else is an extension of the form (*) with $\operatorname{len}(W)=\operatorname{len}(Z) \leq d$. By the second part of that theorem, any sheaf $i_{*} L_{C}$ or extension of the form $(*)$ with len $(W)=\operatorname{len}(Z)<d$ that is $t_{d}-\epsilon$-stable is also $t_{d}+\epsilon$-stable. This just leaves the points of $P^{\prime}$, which are all $t_{d}-\epsilon$-stable, by the same argument as in Step 1. Thus the points of $\mathcal{M}^{\prime}$ are in bijection with the isomorphism classes of all the $t_{d}-\epsilon$-stable objects of $\mathcal{A}^{\#}$ (with the given invariants)!

Proof of Step 2. Let $\sigma: \tilde{\mathcal{M}} \rightarrow \mathcal{M}$ be the blow-up along $\mathbb{P}_{d}$, and let

$$
\tilde{\mathcal{U}}:=L \sigma^{*} \mathcal{U}
$$

be the (derived) pull-back of the coherent sheaf $\mathcal{U}$ from $S \times \mathcal{M}$ to $S \times \tilde{\mathcal{M}}$. We will prove that $\widetilde{U}$ is in fact a coherent sheaf, and then construct $\mathcal{U}^{\prime}$ by descending a (generalized) elementary modification of $\widetilde{\mathcal{U}}$. Carrying out this elementary modification will require two (!) applications of the octahedral axiom.

Lemma 5.2. Suppose $\mathcal{E}_{X}$ is a flat family of objects of $\mathcal{A}^{\#}$ over a connected quasi-projective base $X$. If $\mathcal{E}_{x}$ is (quasi-isomorphic to) a coherent sheaf on $S$ for some closed point $x \in X$, then $\mathcal{E}_{X}$ is (quasi-isomorphic to) a coherent sheaf on $S \times X$. 
Proof. The object $\mathcal{E}_{X}$ can be represented (in $\mathcal{D}^{b}(S \times X)$ ) by a two-term complex $[A \rightarrow B]$. Moreover, by pulling back under a surjective map $V \rightarrow B$, we may assume, without loss of generality, that $B=V$ is a locally free sheaf. Since $\varepsilon_{X}$ is a flat family of objects of $\mathcal{A}^{\#}$, in particular we know that each restriction $\mathcal{E}_{X}$ has cohomology only in degrees -1 and 0 . Thus it follows that $A$ is flat as a coherent sheaf over $X$. Now suppose that some $\varepsilon_{x}$ is a coherent sheaf on $S$. Then the kernel of the map $A \rightarrow V$ must be zero generically over $X$, and, if non-zero, would determine an embedded point of $A$ that does not dominate $X$. Such coherent sheaves are not flat over $X$. Thus $A \rightarrow V$ is injective, and $\mathcal{E}_{X}$ is (quasiisomorphic to) a coherent sheaf.

Corollary 5.3. The flat families $\mathcal{E}_{d}$ and $L i^{*} \mathcal{U}$ on $S \times \mathbb{P}_{d}$ are (quasi-isomorphic to) coherent sheaves.

Proof. Among the extensions parametrized by $\varepsilon_{d}$ are the extensions yielding

$$
0 \rightarrow \mathcal{O}_{S} \rightarrow \mathcal{J}_{Z}(H) \rightarrow \mathcal{O}_{C}(H+W-Z) \rightarrow \mathcal{O}_{W} \rightarrow 0
$$

where $C \in|H|$ is a smooth curve, and $W, Z \subset C$ are disjoint effective divisors of degree $d$ on $C$ (see the example preceding Lemma 3.6). Such an extension $\epsilon$ defines $\mathcal{O}_{C}(H+W-Z)$ as $\left(\mathcal{E}_{d}\right)_{\epsilon}$.

Consider now the pair of coherent sheaves on $S \times\left.\mathbb{P}_{d} \mathcal{U}\right|_{S \times \mathbb{P}_{d}}$ and $\mathcal{E}_{d}$. We cannot conclude that the two sheaves are isomorphic, because there is a built-in ambiguity from the fact that $\mathcal{U}$ and $\mathcal{U} \otimes \mathcal{L}_{\mathcal{M}}$ give equivalent universal families for any line bundle $\mathcal{L}_{\mathcal{M}}$ on $\mathcal{M}$ (this is, as in the case of the Jacobian, the only ambiguity). But we can "match" them as closely as we need with the following:

Lemma 5.4. The map $\operatorname{Pic}(\mathcal{M}) \rightarrow \mathbb{Z}=\operatorname{Pic}\left(\mathbb{P}_{d}\right) / \operatorname{Pic}(S[d] \times S[d])$ is surjective.

Proof. For fixed disjoint reduced subschemes $W, Z \subset S$ of length $d$, the fiber of $\mathbb{P}_{d}$ over the points $(Z, W)$ is, outside a subvariety of codimension $>1$, isomorphic to the linear series $\mathbb{P}^{g-2 d}=\left|\mathcal{O}_{S}(H) \otimes \mathcal{J}_{Z} \otimes \mathcal{J}_{W}\right|$ of curves passing through the points of $Z$ and $W$ (determining the line bundle $\mathcal{O}_{C}(H+W-Z)$ as in the proof of the corollary above). The line bundle $\pi^{*} \mathcal{O}_{\mathbb{P} g}(1)$ pulled back from the "linear series map" $\pi: \mathcal{M}_{S}(0, H, g-1) \rightarrow \mathbb{P}^{g}$ carries over to a line bundle on $\mathcal{M}$ (across all previous Mukai flops), which agrees with $\mathcal{O}_{\mathbb{P}^{g-2 d}}(1)$ off codimension $>1$. Thus this line bundle on $\mathcal{M}$ generates the relative Picard group of $\mathbb{P}_{d}$ over $S[d] \times S[d]$, as desired.

Corollary 5.5. There is a choice of "Poincaré" coherent sheaf $\mathcal{U}$ on $S \times \mathcal{M}$ and line bundle $\mathcal{L}$ on $S[d] \times S[d]$ such that $\left.\mathcal{U}\right|_{S \times \mathbb{P}_{d}} \cong \mathcal{E}_{d} \otimes \mathcal{L}$. Hence, $\left.\mathcal{U}\right|_{S \times \mathbb{P}_{d}}$ fits in a distinguished triangle of the form

$$
\left.\cdots \rightarrow \rho^{*}\left(\mathcal{J}_{Z_{12}}(H) \otimes \mathcal{L}\right) \rightarrow \mathcal{U}\right|_{S \times \mathbb{P}_{d}} \stackrel{u}{\rightarrow} \rho^{*}\left(\mathcal{J}_{Z_{13}}^{\vee}[1] \otimes \mathcal{L}\right) \otimes \mathcal{O}_{\mathbb{P}_{d}}(-1) \rightarrow \cdots
$$

$\left(\rho: S \times \mathbb{P}_{d} \rightarrow S \times S[d] \times S[d]\right.$ and other notation from the proof of Proposition 4.2). 
Proof. By Lemma 5.4, we can match $\left.\mathcal{U}\right|_{S \times \mathbb{P}_{d}}$ and $\mathcal{E}_{d}$ up to the twist of a line bundle $\mathcal{L}$ pulled back from $S[d] \times S[d]$. The distinguished triangle is then the corresponding twist of the distinguished triangle defining $\mathcal{E}_{d}$ in the proof of Proposition 4.2.

Now, let $i_{D}: D \hookrightarrow \tilde{\mathcal{M}}$ be the exceptional divisor of the blow-up, and let $D_{S}=S \times D \subset$ $S \times \widetilde{\mathcal{M}}$ with projections $p: D_{S} \rightarrow S \times \mathbb{P}_{d}$ and $p^{\prime}: D_{S} \rightarrow S \times \mathbb{P}_{d}^{\vee}$. Then we define $\tilde{\mathcal{U}}^{\prime} \in \mathcal{D}(S \times \tilde{\mathcal{M}})$ via the distinguished triangle

$$
\cdots \rightarrow \tilde{\mathcal{U}}^{\prime} \rightarrow \tilde{\mathcal{U}} \stackrel{u \circ r}{\longrightarrow} i_{D_{S *}} p^{*}\left(\rho^{*}\left(\mathcal{J}_{Z_{13}}^{\vee}[1] \otimes \mathcal{L}\right) \otimes \mathcal{O}_{\mathbb{P}_{d}}(-1)\right) \rightarrow \tilde{\mathcal{U}}^{\prime}[1] \rightarrow \cdots
$$

where the "restriction map" $r$ fits in the distinguished triangle (of coherent sheaves!)

$$
\left.\cdots \rightarrow \tilde{\mathcal{U}}\left(-D_{S}\right) \rightarrow \tilde{\mathcal{U}} \stackrel{r}{\rightarrow} i_{D_{S} *} \tilde{\mathcal{U}}\right|_{D_{S}} \rightarrow \tilde{\mathcal{U}}\left(-D_{S}\right)[1] \rightarrow \cdots
$$

and $u$ is (by abuse of notation) the map from the distinguished triangle of Corollary 5.5, pulled back to $D_{S}$ and pushed forward to $S \times \tilde{\mathcal{M}}$. The octahedral axiom applied to the morphisms $u$ and $r$ now produces a distinguished triangle

$$
\cdots \rightarrow \tilde{U}\left(-D_{S}\right) \rightarrow \tilde{U}^{\prime} \stackrel{v}{\rightarrow} i_{D_{S *}} p^{*} \rho^{*}\left(\mathcal{J}_{z_{12}}(H) \otimes \mathcal{L}\right) \rightarrow \tilde{U}\left(-D_{S}\right)[1] \rightarrow \cdots
$$

Remark. This is the derived category version of an elementary modification of a coherent sheaf by a quotient sheaf supported on a divisor. Although the object we are modifying, $\widetilde{\mathcal{U}}$, is indeed a coherent sheaf, it is being modified by a "quotient" which is not a coherent sheaf. Nevertheless, we constructed the modified object $\widetilde{\mathcal{U}}$ ' as the (shifted) cone of the morphism to the "quotient" object supported on $D_{S}$.

Next, we claim that the (derived) restriction of $\widetilde{U}^{\prime}$ to $D_{S}$ satisfies

$$
L i_{D_{S}}^{*} \tilde{U}^{\prime} \cong p^{*} \mathcal{F}_{d} \otimes \mathcal{L}
$$

where $\mathcal{F}_{d}$ is the "universal extension" on $S \times \mathbb{P}_{d}^{\vee}$ from Proposition 4.2,

$$
\cdots \rightarrow\left(\rho^{\vee}\right)^{*}\left(\mathcal{J}_{\mathcal{Z}_{13}}^{\vee}[1]\right) \otimes \mathcal{O}_{\mathbb{P}_{d}^{\vee}}(+1) \rightarrow \mathcal{F}_{d} \rightarrow\left(\rho^{\vee}\right)^{*}\left(\mathcal{J}_{Z_{12}}(H)\right) \rightarrow \cdots
$$

Note that $\mathcal{F}_{d}$ (and its pull-back) is definitely not a coherent sheaf, although $\tilde{\mathcal{U}}^{\prime}$ itself will be. We see the claim with another application of the octahedral axiom, to the two morphisms: $\tilde{\mathcal{U}}^{\prime} \stackrel{r}{\rightarrow} i_{D_{S *}} L i_{D_{S}}^{*} \tilde{U}^{\prime}$ and the push-forward of

$$
L i_{D_{S}}^{*} \tilde{U}^{\prime} \stackrel{v}{\rightarrow} p^{*} \rho^{*}\left(\mathcal{J}_{Z_{12}}(H) \otimes \mathcal{L}\right) \cong p^{*}\left(\rho^{\vee}\right)^{*}\left(\mathcal{J}_{Z_{12}}(H) \otimes \mathcal{L}\right)
$$

( $v$ is the left adjoint of the map $v$ defined by $(* *)$ ). Let $K$ be defined by the distinguished triangle

$$
\cdots \rightarrow K \rightarrow L i_{D_{S}}^{*} \tilde{U}^{\prime} \stackrel{v}{\rightarrow} p^{*} \rho^{*}\left(\mathcal{J}_{Z_{12}}(H) \otimes \mathcal{L}\right) \rightarrow K[1] \rightarrow \cdots .
$$

Then the octahedral axiom applied to $r$ and $i_{D_{S *}} v$ gives

$$
\cdots \rightarrow \tilde{U}^{\prime}\left(-D_{S}\right) \rightarrow \tilde{U}\left(-D_{S}\right) \rightarrow i_{D_{S *}} K \rightarrow \cdots
$$


Combining this with $(*)$ (tensored by $\mathcal{O}\left(-D_{S}\right)$ ), using the projection formula, the faithfulness of $i_{D_{S *}}$ and the isomorphism $\mathcal{O}_{D_{S}}\left(-D_{S}\right) \cong p^{*} \mathcal{O}_{\mathbb{P}_{d}}(1) \otimes p^{\prime *} \mathcal{O}_{\mathbb{P}_{d}^{\vee}}(1)$ gives the desired isomorphism

$$
\begin{aligned}
K & \cong p^{*}\left(\rho^{*}\left(\mathcal{J}_{\mathcal{Z}_{13}}^{\vee}[1] \otimes \mathcal{L}\right) \otimes \mathcal{O}_{\mathbb{P}_{d}}(-1)\right) \otimes \mathcal{O}_{D_{S}}\left(-D_{S}\right) \\
& \cong p^{\prime *}\left(\left(\rho^{\vee}\right)^{*}\left(\mathcal{J}_{\mathcal{Z}_{13}}^{\vee}[1] \otimes \mathcal{L}\right) \otimes \mathcal{O}_{\mathbb{P}_{d}^{\vee}}(+1)\right) .
\end{aligned}
$$

Main Point of Step 2: There is a coherent sheaf $\mathcal{U}^{\prime}$ on $S \times \mathcal{M}^{\prime}$ which is a flat family of objects of $\mathcal{A}^{\#}$ such that the restrictions $\left.\mathfrak{U}^{\prime}\right|_{S \times\left\{m^{\prime}\right\}}$ are in bijection with the set of all $t_{d}-\epsilon$-stable objects of $\mathcal{A}^{\#}$ (with the given invariants). This coherent sheaf is obtained by descending the object $\widetilde{U}^{\prime} \in \mathcal{D}(S \times \widetilde{\mathcal{N}})$, defined above, to $\mathcal{D}\left(S \times \mathcal{M}^{\prime}\right)$. That is,

$$
L_{\sigma^{\prime}}^{*} \mathfrak{U}^{\prime} \cong \widetilde{U}^{\prime}
$$

Proof. Since $\widetilde{\mathcal{U}}^{\prime}$ and $\widetilde{\mathcal{U}}$ coincide away from the exceptional divisor $D_{S}$, and $L i_{D_{S}} * \widetilde{\mathcal{U}}^{\prime}$ is the pull-back of a (universal) family of extensions of objects of $\mathcal{A}^{\#}$, it follows that $\widetilde{U}^{\prime}$ is a flat family of objects of $\mathcal{A}^{\#}$. Moreover, as in Corollary 5.3, it follows that $\widetilde{\mathcal{U}}^{\prime}$ is a coherent sheaf, and that if $\widetilde{\mathcal{U}}^{\prime}=L_{\sigma^{\prime}}^{*} \mathcal{U}^{\prime}$ for some object $\mathcal{U}^{\prime} \in \mathcal{D}\left(S \times \mathcal{M}^{\prime}\right)$, then $\mathcal{U}^{\prime}$ is a coherent sheaf as well. The fact that $\mathcal{U}^{\prime}$ then parametrizes all $t_{d}-\epsilon$-stable objects was established in the Interlude above. But, as explained to us by Andrei Căldăraru [Cald], the descent is an immediate consequence of Orlov's semi-orthogonal decomposition of the derived category of the blow-up $\sigma^{\prime}$ [Or192]. Indeed, it follows from the decomposition that any object $\mathcal{E} \in \mathcal{D}(S \times \widetilde{\mathcal{M}})$ whose restriction to the exceptional divisor descends in the derived category (i.e. $L i_{D_{S}}{ }^{*} \mathcal{E} \cong \sigma^{*} \mathcal{F}$ for some $\left.\mathcal{F} \in \mathcal{D}\left(S \times \mathbb{P}_{d}^{\vee}\right)\right)$ must itself descend.

Proof of Step 3. To recap, the moduli functor

\{flat families of $t_{d}-\epsilon$-stable objects of $\mathcal{A}^{\#}$ with invariants $\left.\left(0, H, H^{2} / 2\right)\right\} /$ iso

has the following properties:

- the stable objects of $\mathcal{A}^{\#}$ have only the automorphisms $\mathbb{C}^{*} \cdot$ id,

- the proper variety $\mathcal{N}^{\prime}$ is in bijection with the set of stable objects, and

- the coherent sheaf $\mathcal{U}^{\prime}$ on $S \times \mathcal{M}^{\prime}$ realizes this bijection.

We want to conclude that $\mathcal{M}^{\prime}$ is a (fine) moduli space representing the functor, and $\mathcal{U}^{\prime}$ is a (universal) Poincaré object, which is well-defined up to tensoring by a line bundle pulled back from $\mathcal{M}^{\prime}$. This will follow once we establish the a priori existence of an Artin stack for the functor: flat families of $t$-stable objects of $\mathcal{A}^{\#}$ (with the given invariants, for each $\left.t>1 / 6, t \neq t_{d}\right)$.

First, we appeal to Example A.4(2) of the Appendix to conclude that the functor \{flat families of objects of $\mathcal{A}^{\#}$ with invariants $\left.\left(0, H, H^{2} / 2\right)\right\}$ is an Artin stack, which we will denote by $\mathfrak{M}_{\mathcal{A}^{\#}}\left(0, H, H^{2} / 2\right)$. Step 3 is complete once we show that $t$-stability is an open condition on this functor, hence itself represented by an open substack. To this end, we prove first that $t$-stability for some $t>1 / 6$ is an open condition.

Suppose $B$ is a quasi-projective base scheme, and $\mathcal{E} \in \mathcal{D}(S \times B)$ is a flat family of objects of $\mathcal{A}^{\#}$ with the given invariants. We may assume without loss of generality (see 
the proof of Lemma 5.2 above) that $\mathcal{E}$ is represented by a two-term complex $f: K \rightarrow V$ where $K, V$ are coherent sheaves on $S \times B$ such that $V$ is locally free and $K$ is flat (as a coherent sheaf) over $B$. By Theorem 3.7, if $\mathcal{E}_{S \times\{b\}}$ is $t$-stable for some closed point $b \in B$ and $t>1 / 6$, then either $f_{b}:=\left.f\right|_{S \times\{b\}}: K_{b} \rightarrow V_{b}$ is injective and $\operatorname{coker}\left(f_{b}\right) \cong i_{*} L_{C}$, or else $E_{b}=\left[K_{b} \stackrel{f}{\rightarrow} V_{b}\right]$ fits in a short exact sequence (in $\mathcal{A}^{\#}$ ) of the form $0 \rightarrow \mathcal{J}_{W}^{\vee}[1] \rightarrow$ $E_{b} \rightarrow \mathcal{J}_{Z}(H) \rightarrow 0$. In the latter case, it follows in particular that $f_{b}$ fits in a long exact sequence

$$
0 \rightarrow \mathcal{O}_{S} \rightarrow K_{b} \stackrel{f_{b}}{\rightarrow} V_{b} \rightarrow Q \rightarrow 0
$$

where $Q$ is a coherent sheaf fitting into $0 \rightarrow \mathcal{O}_{W}^{\vee}[2] \rightarrow Q \rightarrow \mathcal{J}_{Z}(H) \rightarrow 0$.

A little analysis gives the following necessary conditions for $E_{b}$ to be $t$-stable for some $t>1 / 6$ :

(a) $K_{b}$ is locally free (and then it follows that $\operatorname{ker}\left(f_{b}\right)$ is locally free).

(b) $\operatorname{ker}\left(f_{b}\right)=H^{-1}\left(E_{b}\right)$ has rank $\leq 1$, and $c_{1}\left(\operatorname{ker}\left(f_{b}\right)\right) \leq 0$.

(c) len(tors $\left.\left(\operatorname{coker}\left(f_{b}\right)\right)\right)<H^{2} / 8$.

On the other hand, (a)-(c) are very nearly sufficient conditions for $E_{b}$ to be $t$-stable for some $t>1 / 6$. First, if $f_{b}$ is injective, then clearly $E_{b} \cong i_{*} L_{C}$ where $L_{C}$ is torsionfree. If, on the other hand, $\operatorname{ker}\left(f_{b}\right)$ is a line bundle, then by (b), it must be of the form $\mathcal{O}_{S}(-n H)$ for some $n \geq 0$. But $E_{b} \in \mathcal{A}^{\#}$ has invariants $\left(0, H, H^{2} / 2\right)$, by assumption, and this implies that $n=0$, i.e. $\operatorname{ker}\left(f_{b}\right) \cong \mathcal{O}_{S}$ and coker $\left(f_{b}\right)$ has torsion only in dimension zero. Moreover, $\operatorname{coker}\left(f_{b}\right) /$ tors $\cong \mathcal{J}_{Z}(H)$ for some $Z \subset S$ of length equal to the length of the torsion (hence less than $H^{2} / 8$ ), and as in Lemma 3.4, one may conclude that the kernel (in $\mathcal{A}^{\#}$ ) of the map $E_{b} \rightarrow \operatorname{coker}\left(f_{b}\right) /$ tors is of the form $\mathcal{J}_{W}^{\vee}[1]$. Such extensions are necessarily $t$-stable for $t$ very close to the value $t_{d}$, where $d=\operatorname{len}(Z)$, provided that they are non-split. Thus to ensure stability for some $t>1 / 6$, we need only add:

(d) $E_{b}$ is not in the image of any of the (proper) morphisms $h_{d}: S[d] \times S[d] \rightarrow$ $\mathfrak{M}_{\mathcal{A}^{\#}}\left(0, H, H^{2} / 2\right) ;(W, Z) \mapsto \mathcal{J}_{W}^{\vee}[1] \oplus \mathcal{J}_{Z}(H)$ for any $d<H^{2} / 8$.

Finally, suppose $t_{d_{0}}<t<t_{d_{0}-1}$. Then $E_{b}$ is $t$-stable if it is $t$-stable for some $t>1 / 6$, and moreover, it avoids both the images of the (proper) morphisms from Proposition 4.2:

$$
i_{d}: \mathbb{P}_{d} \rightarrow \mathfrak{M}_{\mathcal{A}^{\#}}\left(0, H, H^{2} / 2\right) \quad \text { defined by } \mathcal{E}_{d} \text { for } d<d_{0}
$$

and

$$
i_{d}^{\prime}: \mathbb{P}_{d}^{\vee} \rightarrow \mathfrak{M}_{\mathcal{A}^{\#}}\left(0, H, H^{2} / 2\right) \quad \text { defined by } \mathcal{F}_{d} \text { for } d \geq d_{0}
$$

This completes the proof of Step 3, and hence of Theorem 5.1.

Remark. It certainly ought to be possible to extend this theorem to remove the $t>$ $1 / 6$ assumption, i.e. to produce Mukai flops for "higher rank" walls, in addition to the rank one walls. There are two places where improvements would need to be made to the proof. First, our argument in Step 3 for the openness of stability breaks down when higher rank walls are crossed (but a very recent result of Toda [Tod07] gives an independent proof of the existence of the Artin stack). Second, there are cases where higher rank 
walls coincide (with each other or with a rank one wall). In that case, the wall-crossing will not be a simple Mukai flop, but more likely a stratified elementary modification of the sort investigated by Markman [Mar01] for the birational transformations of moduli spaces induced by Fourier-Mukai transforms. In any case, this theorem is quite likely to generalize in many interesting ways.

\section{K3 surfaces}

Let $S$ be a K3 surface of genus $g$ with $\operatorname{Pic}(S)=\mathbb{Z}[H]$ (i.e. $H^{2}=2 g-2$ ). The results of $\S 4$ and $\S 5$ then give the following:

Vanishing: For all $d<H^{2} / 8=\frac{1}{4}(g-1)$ and all pairs $Z, W \subset S$ of closed subschemes of length $d$,

$$
H^{i}\left(S, \mathcal{J}_{W} \otimes \mathcal{J}_{Z}(H)\right)=0 \quad \text { for all } i>0
$$

(Proposition 4.1).

Moduli spaces: By Theorem 5.1, there are Mukai flops of the relative Jacobian

$$
\mathcal{M}_{S}\left(0, H, H^{2} / 2\right)=\mathcal{M}_{0} \rightarrow \mathcal{M}_{1--\rightarrow \cdots-\rightarrow} \mathcal{M}_{d_{g}}
$$

as one crosses walls $t_{d}:=\sqrt{1 / 4-d \cdot 2 / H^{2}}>1 / 6$. Thus, the final flop predicted by the theorem finishes with $\mathcal{M}_{d_{g}}$ where $d_{g}=\left\lceil H^{2} / 9\right\rceil$ is the round-up of $H^{2} / 9=(2 g-2) / 9$.

However, these results for K3 surfaces are not optimal. For example, the vanishing theorem predicts that $\mathcal{O}_{S}(H)$ is very ample on a $\mathrm{K} 3$ surface of genus $\geq 6$, whereas in fact it is very ample for genus $\geq 3$. We can get better results if we use Bridgeland's central charge:

$$
Z_{(D, F)}^{\prime}(E):=-\int_{S} e^{-(D+i F)} \operatorname{ch}([E]) \sqrt{\operatorname{td}(S)}=Z_{(D, F)}(E)-\operatorname{rk}(E)
$$

instead of the central charge (without the Todd class contribution) $Z_{(D, F)}$ of $\S 2$. The key point is that if $E$ is an $H$-stable vector bundle on a K3 surface $S$, then $\chi\left(S, E \otimes E^{*}\right) \leq 2$ and a quick computation with Riemann-Roch shows that

$$
\operatorname{ch}_{2}(E) \leq \frac{c_{1}^{2}(E)}{2 \cdot \operatorname{rk}(E)}-\operatorname{rk}(E)+\frac{1}{\operatorname{rk}(E)}
$$

(for all $H$-stable torsion-free sheaves), which is sharper than the Bogomolov-Gieseker inequality. For the choice $Z_{t}^{\prime}:=Z_{\left(\frac{1}{2} H, t H\right)}^{\prime}$ and category $\mathcal{A}^{\#}$ as before, we now get the following version of Corollary 2.1:

Corollary 6.1. On a K3 surface $S$ of genus $g$ and Picard number one, $\left(Z_{t}^{\prime}, \mathcal{A}^{\#}\right)$ is a Bridgeland slope function if either $g$ is odd and $t>0$, or else $g$ is even and $t>$ $\sqrt{1 /(4 g-4)}$. 
Proof. The proof of Corollary 2.1 holds up until the final case:

$$
\mu_{t H}(E)=\left(\frac{1}{2} H\right) \cdot(t H) \Leftrightarrow \mu_{H}(E)=\frac{c_{1}(E) \cdot H}{\operatorname{rk}(E)}=\frac{1}{2} H^{2},
$$

which, because of the Picard number one assumption, implies that

$$
c_{1}(E)=c H, \quad \operatorname{rk}(E)=2 c \quad \text { for some integer } c .
$$

For torsion-free sheaves with these invariants, we then use the improved inequality to obtain

$$
\operatorname{Re}\left(Z_{t}^{\prime}(E)\right) \geq t^{2} \operatorname{rk}(E) \frac{H^{2}}{2}-\frac{1}{\operatorname{rk}(E)}=t^{2} c H^{2}-\frac{1}{2 c},
$$

and this is positive if $t^{2}\left(H^{2}\right)>1 / 2$ or $t>\sqrt{1 /(4 g-4)}$, giving the result for even genera.

When $g$ is odd and $r=2 c$ is even, the inequality can be improved:

$$
\operatorname{ch}_{2}(E) \leq \frac{g-1}{2 c}-2 c+\frac{1}{2 c} \Rightarrow \operatorname{deg}\left(\operatorname{ch}_{2}(E)\right) \leq \frac{g-1}{2 c}-2 c
$$

because $\operatorname{deg}\left(\operatorname{ch}_{2}(E)\right)$ is an integer (!). This gives the better result for odd genera.

Lemma 3.1 remains valid (with the same proof!) for $Z_{t}^{\prime}$ and we obtain the following:

Proposition 6.2 (Better vanishing). For a $K 3$ surface $S$ of genus $g$ with $\operatorname{Pic}(S)=\mathbb{Z}[H]$,

$$
H^{i}\left(S, \mathcal{J}_{W} \otimes \mathcal{J}_{Z}(H)\right)=0 \text { for all } i>0 \text { and all subschemes } Z, W \text { of length } d<\frac{g+2}{4} \text {. }
$$

Proof. As in the proof of Proposition 4.1, it suffices to exhibit a value of $t$ such that $\left(Z_{t}^{\prime}, \mathcal{A}^{\#}\right)$ is a Bridgeland slope function (i.e. satisfies the criteria of Corollary 6.1) and

$$
\mu_{t}^{\prime}\left(\mathcal{J}_{W}^{\vee}[1]\right)=0=\mu_{t}^{\prime}\left(\mathcal{J}_{Z}(H)\right)
$$

where $\mu^{\prime}=-\operatorname{Re}\left(Z^{\prime}\right) / \operatorname{Im}\left(Z^{\prime}\right)$. Solving this equality yields

$$
d=\frac{H^{2}}{8}-\frac{t^{2} H^{2}}{2}+1=\frac{g+3}{4}-t^{2}(g-1)
$$

and keeping mind the constraints on $t$, this yields $d<(g+3) / 4$ if $g$ is odd, and $d<$ $(g+2) / 4$ if $g$ is even. Since $d$ must be an integer, the result of the proposition is then sharp.

Remark. This bound is now the best possible, since, for example, it gives very ampleness of $\mathcal{O}_{S}(H)$ in genus 3, but just fails to give it in genus 2 (where $\mathcal{O}_{S}(H)$ is NOT very ample!). 
To get the right number of flops on a K3 surface, note that the flops will exist for all the critical values

$$
t=\sqrt{\frac{(g+3) / 4-d}{g-1}}
$$

until $t$ runs into the first "higher (odd) rank wall," (the value $t=1 / 6$ in the proof of Theorem 3.7). As in that theorem, this highest value of $t$ is computed by setting

$$
\operatorname{Re}\left(Z_{t}^{\prime}(E)\right)=0
$$

where $E$ is an $H$-stable torsion-free sheaf with $\operatorname{rk}(E)=3, c_{1}(E)=2 H$, and $\operatorname{ch}_{2}(E)$ maximal. Using the inequality

$$
\operatorname{deg}\left(\operatorname{ch}_{2}(E)\right) \leq \frac{(2 H)^{2}}{6}-3+\frac{1}{3}=\frac{4 g}{3}-4
$$

and the fact that it is an integer, we obtain (sharp) values for such $t$ by setting:

$$
0= \begin{cases}-\frac{1}{24} H^{2}+\frac{3}{2} t^{2} H^{2}-\frac{1}{3} & \text { if } g=3 n \\ -\frac{1}{24} H^{2}+\frac{3}{2} t^{2} H^{2} & \text { if } g=3 n+1, \\ -\frac{1}{24} H^{2}+\frac{3}{2} t^{2} H^{2}+\frac{1}{3} & \text { if } g=3 n+2 .\end{cases}
$$

It follows that in all genera $>2$, the upper bound on the number of Mukai flops improves to

$$
d_{g}=\lceil 2(g+3) / 9\rceil \text {. }
$$

Small genus examples: Genus 2. In all genera $\geq 2$, the first Mukai flop exists:

$$
\mathcal{M}_{0}-\rightarrow \mathcal{M}_{1}
$$

replacing $\mathbb{P}^{g}:=\mathbb{P}\left(H^{0}\left(S, \mathcal{O}_{S}(H)\right)\right)$ with its dual $\left(\mathbb{P}^{g}\right)^{\vee}=\mathbb{P}\left(\operatorname{Ext}_{S}^{2}\left(\mathcal{O}_{S}(H), \mathcal{O}_{S}\right)\right)$.

This Mukai flop can also be realized with the (standard) Fourier-Mukai transform

$$
i_{*} L_{C} \mapsto R \pi_{2 *}\left(R \pi_{1}^{*} i_{*} L_{C} \stackrel{L}{\otimes} \mathcal{J}_{\Delta}\right)^{\vee}
$$

where $\pi: S \times S \rightarrow S$ are the projections, and $\Delta \subset S \times S$ is the diagonal.

We claim that, in fact, $\mathcal{M}_{1} \cong \mathcal{M}_{S}\left(g-1, H,-H^{2} / 2\right)$. Here is a sketch of the argument, which is well-known [Mar01]. Consider first the case $L_{C} ¥ \omega_{C}$. Then $\operatorname{dim}\left(H^{0}\left(S, i_{*} L_{C}\right)\right)$ $=g-1$, and

$$
E=\left[H^{0}\left(S, i_{*} L_{C}\right) \otimes \mathcal{O}_{S} \stackrel{r}{\rightarrow} i_{*} L_{C}\right]^{\vee}
$$

is a stable torsion-free sheaf with the desired invariants (which is locally free iff $r$ is surjective). This gives a rational map $\Phi: \mathcal{M}_{0}-\rightarrow \mathcal{M}_{S}\left(g-1, H,-H^{2} / 2\right)$ that is welldefined off the locus $\mathbb{P}^{g}$ parametrizing the sheaves of the form $i_{*} \omega_{C}$. To define $\Phi$ further, we blow up $\mathbb{P}^{g} \subset \mathcal{M}_{0}$ by choosing codimension one subspaces $V \subset H^{0}\left(C, \omega_{C}\right)$ at each point $i_{*} \omega_{C} \in \mathbb{P}^{g}$. Each such point is then mapped to $\left[V \otimes \mathcal{O}_{S} \rightarrow i_{*} \omega_{C}\right]^{\vee}$, extending $\Phi$ to $\widetilde{\mathcal{M}}$, and furthermore one can check that the map $\Phi$ blows down the exceptional divisor $D$, 
giving the Mukai flop. Notice that in the genus 2 case, $\mathcal{M}_{S}\left(1, H,-H^{2} / 2\right) \cong S[2]$ is the Hilbert scheme parametrizing sheaves of the form $\mathcal{J}_{Z}(H)$.

Genus 3. In all genera $\geq 3$, there is a second Mukai flop

$$
\mathcal{M}_{1} \rightarrow \mathcal{M}_{2} \text {. }
$$

In genus 3 , the flop locus $\mathbb{P}_{1} \subset \mathcal{M}_{1}$ is a divisor (codimension $3-2 \cdot 1=1$ ). Thus in genus 3 , this second "flop," which occurs at $t=1 / 2$ (corresponding to $d=1$ ) is actually the identity. On the other hand, there is one further "rank three wall" at $t=\sqrt{1 / 12}$, and the indeterminacy locus for that wall is, again, $\left(\mathbb{P}^{g}\right)^{\vee}$ so that the rank three wall is the inverse of the original flop (!). That is, there is a symmetry:

$$
\mathcal{M}_{0} \text { 1st rank one flop } \mathcal{M}_{1} \stackrel{\text { 2nd rank one flop }}{\cong} \mathcal{M}_{1} \stackrel{\text { rank } 3 \text { flop }}{\cong} \mathcal{M}_{0}
$$

Experimental evidence suggests some sort of similarly symmetric picture when all the higher rank walls are taken into account in each genus of the form $4 n+3$.

\section{Abelian surfaces}

Let $S$ be a simple abelian surface, with $(1, D)$ polarization and $\mathrm{NS}(S) \cong Z[H]$, so

$$
H^{2}=2 D \quad \text { and } \quad h^{0}\left(S, \mathcal{O}_{S}(H)\right)=D .
$$

Then the vanishing and main theorem hold in the following modified forms:

Vanishing: For all subschemes $Z, W \subset S$ of length $<H^{2} / 8=D / 4$ and all $\mathcal{L} \in$ $\operatorname{Pic}^{0}(S)$

$$
\mathrm{H}^{i}\left(S, \mathcal{J}_{W} \otimes \mathcal{J}_{Z}(H) \otimes \mathcal{L}\right)=0 \quad \text { for } i=1,2 .
$$

No better vanishing is to be expected, since the Bogomolov-Gieseker bound matches the bound coming from Riemann-Roch applied to

$$
\chi\left(E \otimes E^{*}\right) \leq 2 \quad \text { for } H \text {-stable bundles. }
$$

In this case, as well, $\operatorname{td}(S)=1$, and the two central charges $Z$ and $Z^{\prime}$ coincide.

Moduli: Let $\widehat{S}=\operatorname{Pic}^{0}(S)$ be the dual abelian surface. Then Proposition 4.2 can be easily modified to produce projective bundles $\mathbb{P}_{d}, \mathbb{P}_{d}^{\vee}$ over $(\widehat{S} \times S[d]) \times(\widehat{S} \times S[d])$ parametrizing extensions

$$
\begin{aligned}
& 0 \rightarrow \mathcal{L}_{1} \otimes \mathcal{J}_{Z}(H) \rightarrow E_{d} \rightarrow \mathcal{L}_{2} \otimes \mathcal{J}_{W}^{\vee}[1] \rightarrow 0 \text { and } \\
& 0 \rightarrow \mathcal{L}_{2} \otimes \mathcal{J}_{W}^{\vee}[1] \rightarrow F_{d} \rightarrow \mathcal{L}_{1} \otimes \mathcal{J}_{Z}(H) \rightarrow 0
\end{aligned}
$$

(for $\mathcal{L}_{1}, \mathcal{L}_{2} \in \widehat{S}$ ), which embed

$$
\mathbb{P}_{d} \hookrightarrow \mathcal{M}_{t_{d}+\epsilon} \quad \text { and } \quad \mathbb{P}_{d}^{\vee} \hookrightarrow \mathcal{M}_{t_{d}-\epsilon}
$$

as before, as the centers for the birational transformations. 
Here, the dimension count is

$$
\operatorname{dim}(\mathcal{M})=2-\chi\left(\operatorname{RHom}_{\mathcal{D}(S)}(E, E)\right)=2+H^{2}=2 D+2
$$

and $\mathbb{P}_{d}$ has fiber dimension $D-2 d-1$ over a base of dimension $4 d+4$, which therefore continues to satisfy

$$
(\text { fiber dimension })=(\text { codimension })
$$

the condition for the birational transformation replacing $\mathbb{P}_{d}$ with $\mathbb{P}_{d}^{\vee}$ to be a Mukai flop.

And the number of such flops is $\left\lceil H^{2} / 9\right\rceil=\lceil 2 D / 9\rceil$, as before, as well.

\section{Small values for $D$ :}

- $D=1,2,3,4$. Only the $d=0$ flop exists. In the $D=1$ (principal polarization) case, the map $\mathbb{P}_{0}=\widehat{S} \times \widehat{S} \rightarrow \mathcal{M}$ is an isomorphism.

- $D=5$. Vanishing for $d=1$ gives the sharp (and well-known) result: $H$ (and its translates) are very ample when $D \geq 5$ In this $D=5$ case, the embedding $\mathbb{P}_{1} \hookrightarrow \mathcal{M}$ has codimension two.

\section{Stable pairs}

Recall that for a smooth projective curve $C$ with (ample) line bundle $L$, Serre duality gives

$$
\mathbb{P}:=\mathbb{P}\left(H^{0}\left(C, \omega_{C} \otimes L\right)^{*}\right) \cong \mathbb{P}\left(\operatorname{Ext}_{C}^{1}\left(L, \mathcal{O}_{C}\right)\right),
$$

which is then on the one hand a projective space for extensions

$$
0 \rightarrow \mathcal{O}_{C} \rightarrow E \rightarrow L \rightarrow 0
$$

and on the other, the image for the "linear series map" $\phi_{L \otimes \omega_{C}}: C \rightarrow \mathbb{P}$.

Michael Thaddeus [Tha94] showed that there is a one-parameter family of stability conditions on isomorphism classes of "pairs" $\left(\mathcal{O}_{C} \rightarrow E\right)$ satisfying $\operatorname{det}(E) \cong L$ that exhibit wall-crossing behavior and a sequence of birational moduli spaces

$$
\mathbb{P}=: \mathcal{P}_{0} \rightarrow \mathcal{P}_{1} \rightarrow \mathcal{P}_{2} \rightarrow \cdots \cdots \rightarrow \mathcal{P}_{\lfloor(d-1) / 2\rfloor}, \quad d=\operatorname{deg}(L),
$$

such that:

- $\mathcal{P}_{1}$ is the blow-up of $\mathcal{P}_{0}$ along the embedded curve $C$.

- $\mathcal{P}_{d+1}$ is a "flip" of $\mathcal{P}_{d}$, replacing the proper transform of the secant variety of projective $d$-planes spanned by $d+1$ points of $C$ with a projective bundle $P_{d}^{\vee} \rightarrow \operatorname{Sym}^{d+1}(C)$ with fiber

$$
\mathbb{P}\left(H^{0}\left(C, \omega_{C} \otimes L(-2 D)\right)^{*}\right)=\mathbb{P}\left(\operatorname{Ext}_{C}^{1}\left(L(-D), \mathcal{O}_{C}(D)\right)\right) \text { over } D \in \operatorname{Sym}^{d+1}(C) .
$$


In [Bert97], the second author asked whether such a sequence of moduli spaces might also exist for embeddings $\phi_{L \otimes \omega_{C}}: C \rightarrow \mathbb{P}$ by a sufficiently ample line bundle $L$. We remark here that a non-trivial sequence of "Thaddeus-like" flips does indeed exist when $S$ is a simple K3 surface of genus $g$, by restricting the chain of Mukai flops above

$$
\mathcal{M}_{1 \rightarrow-\rightarrow} \mathcal{M}_{2-\rightarrow \cdots-\rightarrow} \mathcal{M}_{\lceil(2 g+6) / 9\rceil}
$$

to the subvariety

$$
\mathbb{P}_{0}^{\vee}=\mathbb{P}\left(H^{0}\left(S, \mathcal{O}_{S}(H)\right)^{*}\right)=\mathbb{P}\left(\operatorname{Ext}_{S}^{2}\left(\mathcal{O}_{S}(H), \mathcal{O}_{S}\right)\right) \subset \mathcal{M}_{1}
$$

Definition. We will call the proper transform $\mathcal{P}_{d} \subset \mathcal{M}_{d+1}$ of $\mathbb{P}_{0}^{\vee} \subset \mathcal{M}_{1}$ the space of stable pairs for $t$-stable objects $\left(t_{d}<t<t_{d+1}\right)$ with invariants $\left(0, H, H^{2} / 2\right)$.

We now describe candidates for the points of $\mathcal{P}_{d} \subset \mathcal{M}_{d+1}$ (without proof) in a rather close analogy with the case of Thaddeus stable pairs (on curves).

- Each point of $\mathcal{P}_{d}$ represents an object $E \in \mathcal{A}^{\#}$ with a (unique!) non-trivial "section" $\mathcal{O}_{S}[1] \rightarrow E$.

Indeed, every one of the objects parametrized by each $\mathbb{P}_{d}^{\vee}$ fits in an exact sequence

$$
0 \rightarrow \mathcal{J}_{W}^{\vee}[1] \rightarrow E \rightarrow \mathcal{J}_{Z}(H) \rightarrow 0
$$

and thus satisfies $H^{-1}(E)=\mathcal{O}_{S}$, so that the canonical inclusion $H^{-1}(E)[1] \rightarrow E$ is a "section." Moreover, since $\operatorname{Hom}\left(\mathcal{O}_{S}[1], H^{0}(E)\right)=\operatorname{Ext}_{S}^{-1}\left(\mathcal{O}_{S}, H^{0}(E)\right)=0$, it follows that this section is unique (up to scalars). Thus the space of $t$-stable objects $E \in \mathcal{M}_{d}$ admitting sections is the union of the proper transforms of all the $\mathbb{P}_{e}^{\vee} \subset \mathcal{M}_{e+1}$ for all $e<d$. In particular, it contains $\mathcal{P}_{d}$ as a component.

- The quotient by the section, $E / \mathcal{O}_{S}[1]$, satisfies

$$
0 \rightarrow \mathcal{O}_{Z}^{\vee}[2] \rightarrow E / \mathcal{O}_{S}[1] \rightarrow \mathcal{J}_{Z}(H) \rightarrow 0 \quad \text { for some } Z \subset S \text { of length } \leq d .
$$

(Note: $\mathcal{O}_{Z}^{\vee}[2]$ is the torsion coherent sheaf $\mathscr{E} x t_{S}^{2}\left(\mathcal{O}_{Z}, \mathcal{O}_{S}\right)$ of length len $(Z)$ supported on $Z \subset S)$. This is analogous to fixing the determinant $L=\mathcal{O}_{C}(H)$ and noting that the section $\mathcal{O}_{C} \rightarrow E$, if it vanishes along an effective divisor $D \subset C$, gives rise to an exact sequence for the quotient,

$$
0 \rightarrow \mathcal{O}_{D}^{\vee}[1] \rightarrow E / \mathcal{O}_{C} \rightarrow L_{C}(-D) \rightarrow 0
$$

where $\mathcal{O}_{D}^{\vee}[1]$ is the torsion coherent sheaf $\mathscr{E} x t_{S}^{1}\left(\mathcal{O}_{D}, \mathcal{O}_{C}\right)$ of length $\operatorname{deg}(D)$ supported on $D$.

- The sequence above splits. 
This last condition is automatic for curves, by the classification of modules over a PID, but not so in the surface case. Indeed, fixing $Z \subset S$ of length $d+1$, the space of extensions of the form

$$
0 \rightarrow \mathcal{J}_{Z}^{\vee}[1] \rightarrow E \rightarrow \mathcal{J}_{Z}(H) \rightarrow 0
$$

is parametrized by $\mathbb{P}\left(H^{0}\left(S, \mathcal{J}_{Z} \otimes \mathcal{J}_{Z}(H)\right)^{*}\right)$, and one can check that the splitting of the sequence for $H^{0}(E) / \mathcal{O}_{S}[1]$ restricts the extensions to be of the form

$$
H^{0}\left(S, \mathcal{J}_{Z}^{2}(H)\right)^{*} \subset H^{0}\left(S, \mathcal{J}_{Z} \otimes \mathcal{J}_{Z}(H)\right)^{*} .
$$

These are the extensions that would be "inserted" at the $d$ th (Thaddeus) flip.

This is now in perfect analogy with the projective bundle $P_{d}^{\vee} \rightarrow \operatorname{Sym}^{d+1}(C)$ that appears in the Thaddeus flips for curves, though in this case it will not be a projective bundle, since the dimensions of the spaces $H^{0}\left(S, \mathcal{J}_{Z}^{2}(H)\right)$ will jump up.

Final remark. We hope to be able to "fix" this definition by constructing the appropriate moduli problem for stable pairs on a surface (not necessarily K3) in order to define $\mathcal{P}_{d}$ as a moduli space, to determine its scheme structure through deformation theory.

Acknowledgments. We thank Dan Abramovich, Tom Bridgeland, Andrei Căldăraru, Max Lieblich, Dragan Miličić and Alexander Polishchuk for all their help, especially on the subjects of Bridgeland stability conditions and the subtleties of the derived category. We also thank the referee for many useful suggestions.

The first author was partially supported by a faculty research grant from St. Vincent College. The second author was partially supported by NSF grant DMS 0501000.

\section{References}

[AP06] Abramovich, D., Polishchuk, A.: Sheaves of $t$-structures and valuative criteria for stable complexes. J. Reine Angew. Math. 590, 89-130 (2006) Zbl 1093.14026 MR 2208130

[BM09] Bayer, A., Macrì, E.: The space of stability conditions on the local projective plane. Duke Math. J. 160, 263-322 (2011) Zbl 1238.14014 MR 2852118

[Bert97] Bertram, A.: Stable pairs and log flips. In: Algebraic Geometry—Santa Cruz 1995, Proc. Sympos. Pure Math. 62, Part 1, Amer. Math. Soc., 185-201 (1997) Zbl 0961.14020 MR 1492523

[Bri07] Bridgeland, T.: Stability conditions on triangulated categories, Ann. of Math. (2) 166, 317-345 (2007) Zbl 1137.18008 MR 2373143

[Bri08] Bridgeland, T.: Stability conditions on K3 surfaces. Duke Math. J. 141, 241-291 (2008) Zbl 1138.14022 MR 2376815

[Ca105] Căldăraru, A.: Derived categories of sheaves: a skimming. In: Snowbird Lectures in Algebraic Geometry, Contemp. Math. 388, Amer. Math. Soc., Providence, RI, $43-75$ (2005) MR 2182889

[Cald] Căldăraru, A.: private communication

[ES98] Ellingsrud, G., Strømme, A.: An intersection number for the punctual Hilbert scheme of a surface. Trans. Amer. Math. Soc. 350, 2547-2552 (1998) Zbl 0893.14001 MR 1432198

[Fri98] Friedman, R.: Algebraic Surfaces and Holomorphic Vector Bundles, Universitext, Springer, New York (1998) Z Zbl 0902.140291 MR 1600388 
[HRS96] Happel, D., Reiten, I., Smalø, S.: Tilting in abelian categories and quasitilted algebras. Mem. Amer. Math. Soc. 120, no. 575 (1996) Zbl 0849.16011 MR 1327209

[In10] Inaba, M.: Smoothness of the moduli space of complexes of coherent sheaves on an abelian or a projective K3 surface. Adv. Math. 227, 1399-1412 (2011) Zbl 1220.14010 MR 2799799

[Mar01] Markman, E.: Brill-Noether duality for moduli spaces of sheaves on K3 surfaces. J. Algebraic Geom. 10, 623-694 (2001) Zbl 1074.14525 MR 1838974

[Mil] Miličić, D.: Lectures on Derived Categories. http://www.math.utah.edu/milicic/ dercat.pdf

[Muk84] Mukai, S.: Symplectic structure of the moduli space of sheaves on an abelian or K3 surface. Invent. Math. 77, 101-116 (1984) Zbl 0565.14002 MR 0751133

[Or192] Orlov, D. O.: Projective bundles, monoidal transformations and derived categories of coherent sheaves. Izv. Akad. Nauk SSSR Ser. Mat. 56, 852-862 (1992) (in Russian); English transl.: Russian Acad. Sci. Izv. Math. 41, 133-141 (1993) Zbl 0798.14007 MR 1208153

[Tha94] Thaddeus, M.: Stable pairs, linear systems and the Verlinde formula. Invent. Math. 117, 317-353 (1994) Zbl 0882.14003 MR 1273268

[Tod07] Toda, Y.: Moduli stacks and invariants of semistable objects on K3 surfaces. Adv. Math. 217, 2736-2781 (2008) Zbl 1136.14007 MR 2397465

[Ver63] Verdier, J.-L.: Catégories derivées. Quelques résultats. État 0. In: Sém. Géom. Alg. 4 1/2. Cohomologie étale, Lecture Notes in Math. 569, Springer, 262-311 (1977) Zbl 0407.18008

\section{Appendix. The openness of tilted hearts (by Max Lieblich)}

Abstract. We show that there is a natural condition on a torsion theory on the category of coherent sheaves on a flat proper morphism which ensures that the heart of the tilting is represented by an Artin stack locally of finite presentation over the base.

Let $X \rightarrow S$ be a flat proper morphism of finite presentation between schemes. Write $\mathcal{A} \rightarrow S$ for the fpqc stack of quasi-coherent sheaves on $X, \mathcal{A}_{p f}$ for the substack parametrizing quasi-coherent sheaves of finite presentation, and $\mathcal{A}_{p f}^{p}$ for the substack of $\mathcal{A}_{p f}$ consisting of families of quasi-coherent sheaves of finite presentation which are flat over the base. Following a common convention, given a stack (e.g., $\mathcal{A}$ ) and an $S$-scheme $B$, we will use a subscript $B$ to denote the objects of that stack over $B$ (e.g., $\mathcal{A}_{B}$ ). For the moment, we work with the full stacks of categories and not merely the underlying stacks of groupoids. It is a standard result that the stack of groupoids $\left(\mathcal{A}_{p f}^{p}\right)^{\text {gr }}$ underlying $\mathcal{A}_{p f}^{p}$ is an Artin stack locally of finite presentation over $S$. (For the projective case, the reader can consult Theorem 4.6.2.1 in [7]; the general case is handled in §2.1 of [9].)

Remark A.1. Note that while $\mathcal{A}$ is a stack of abelian categories, this is not in fact true of $\mathcal{A}_{p f}$. A simple example which shows that $\mathcal{A}_{p f}$ is not abelian is the following: the homomorphism of finitely presented $\mathbb{Z}\left[x_{1}, x_{2}, \ldots\right]$-modules $\mathbb{Z}\left[x_{1}, x_{2}, \ldots\right] \rightarrow \mathbb{Z}\left[x_{1}, x_{2}, \ldots\right]$ which sends $x_{2 i-1} \mapsto x_{2 i-1}$ and $x_{2 i} \mapsto x_{2 i-1}$ (for $i \geq 1$ ) has kernel $\left(x_{2 i}-x_{2 i-1}\right.$ ), which 
is not even finitely generated. Moreover, this difficulty cannot be avoided by requiring $S$ to be Noetherian, since in the theory of stacks one must allow arbitrary base changes. However, for any field-valued point $s \rightarrow S$, the fiber category $\left(\mathcal{A}_{p f}\right)_{s}$ is abelian: it is the category of coherent sheaves on a finite type $\kappa(s)$-scheme.

Definition A.2. A stack of torsion theories in $\mathcal{A}_{p f}$ consists of a pair of full substacks $(T, F)$ of $\mathcal{A}_{p f}^{p}$ with the property that for each point $s=\operatorname{Spec} K \rightarrow S$, the pair of subcategories $\left(T_{s}, F_{s}\right)$ in $\left(\mathcal{A}_{p f}\right)_{s}$ is a torsion theory in the classical sense of Section 2 above. A stack of torsion theories $(T, F)$ is open if the groupoids underlying $T$ and $F$ are open substacks of $\left(\mathcal{A}_{p f}^{p}\right)^{\mathrm{gr}}$.

By a slight abuse of notation, we will omit the superscripts gr in what follows; when we discuss the openness of a substack, we will always implicitly mean the openness of the associated stack of groupoids in $\mathcal{A}_{p f}^{p}$.

Lemma A.3. Let $(T, F)$ be a stack of torsion theories. Suppose $\operatorname{Spec} K=s \rightarrow S$ is a point and $L / K$ is a field extension. An object $M \in\left(\mathcal{A}_{p f}\right)_{s}$ is in $T_{s}$ if and only if the restriction $M_{\mathrm{Spec} L}$ is in $T_{\mathrm{Spec} L}$, and similarly for $F$.

Proof. The covering Spec $L \rightarrow \operatorname{Spec} K$, together with a choice of clivage of the stacks $\mathcal{A}_{p f}^{p}$ and $T$, yields a diagram of functors

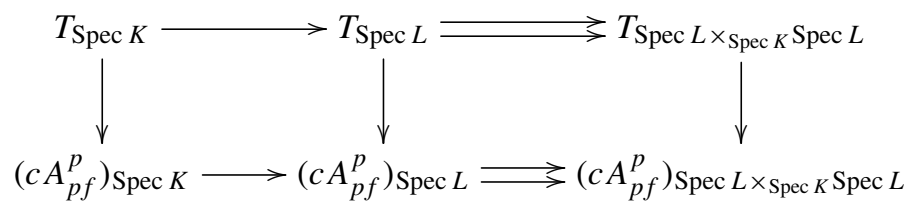

in which the vertical functors are all fully faithful and the horizontal lines exhibit the leftmost categories as the 2-fiber product of the two pullback functors. The full faithfulness of $T \rightarrow \mathcal{A}_{p f}^{p}$ implies that any object of $T_{\mathrm{Spec} L}$ with a descent datum in $\mathcal{A}_{p f}^{p}$ with respect to Spec $L \rightarrow$ Spec $K$ has a canonical descent datum in $T$ with respect to the same covering. It follows that any object of $\left(\mathcal{A}_{p f}^{p}\right)_{\operatorname{Spec} K}$ whose restriction to Spec $L$ lies in $T_{\operatorname{Spec} L}$ comes from a unique object of $T_{\mathrm{Spec} K}$ up to unique isomorphism.

Thus, belonging to $T$ or $F$ is determined by geometric fibers. The torsion theories one encounters "in nature" are often open stacks of torsion theories.

Example A.4. Here are a couple of examples when $X$ is a projective variety over an algebraically closed field.

(1) If $X$ is a smooth projective variety, then letting $T$ be the stack of torsion sheaves and letting $F$ be the stack of torsion free sheaves on $X$ (i.e., pure sheaves on $X$ of maximal dimension) defines an open stack of torsion theories.

(2) If $X$ is a $\mathrm{K} 3$ surface, Bridgeland has described a class of examples (see Lemma 5.1 of [3]). Given an ample divisor $\omega$ on $X$ and a class $\beta \in \mathrm{NS}(X) \otimes \mathbb{R}$, let $T$ be the 
category of coherent sheaves on $X$ whose torsion free parts have the property that all subquotients of the Harder-Narasimhan filtration have slope strictly larger than $\beta \cdot \omega$, and let $F$ be the category of torsion free sheaves on $X$ whose Harder-Narasimhan factors all have slope at most $\beta \cdot \omega$.

Proof that Example A.4(1) defines an open stack of torsion theories. Since the property of being torsion or torsion free on fibers of $X_{B} \rightarrow B$ is determined fpqc-locally on $B$, we see that $T$ and $F$ are full substacks of $\mathcal{A}_{p f}^{p}$. To show that $T$ and $F$ are open in $\mathcal{A}_{p f}^{p}$ we will show that they are constructible and stable under generization. To show this, it suffices to prove it for an arbitrary flat family over an affine scheme $S$. Since membership in $T$ or $F$ is a fiberwise condition and $X$ is of finite type, we may assume that $S$ is itself of finite type over the base field, hence Noetherian.

Let $\mathscr{F}$ be an $S$-flat coherent sheaf on $X_{S}$. Since $S$ is Noetherian, to show that $T$ and $F$ are constructible, it suffices (by the results of $\$ 9.2$ of [4]) to work in the case in which $S$ is irreducible, and show that the generic fiber of $\mathscr{F}$ over $S$ lies in $T$ (resp. $F$ ) if and only if a dense open subset of fibers of $\mathscr{F}$ over $S$ lies in $T$ (resp. $F$ ). We know that the generic fiber $\mathscr{F}_{\eta}$ lies in $T_{\eta}$ if and only if the dimension of the support of $\mathscr{F}_{\eta}$ is strictly less than the dimension of $X$, and that $\mathscr{F}_{\eta}$ is torsion free if and only if $\mathscr{F}_{\eta}$ has no associated point of non-maximal dimension.

Since $\mathscr{F}$ is $S$-flat, its associated points (on the scheme $X_{S}$ ) all lie in the generic fiber $X_{\eta}$, so that the support of $\mathscr{F}$ is the closure of the support of the generic fiber $\mathscr{F}_{\eta}$. The upper semicontinuity of fiber dimension shows that $\mathscr{F}_{\eta}$ is torsion if and only if a dense set of closed fibers of $\mathscr{F}$ over $S$ are torsion, and that if $\mathscr{F}_{\eta}$ contains a non-trivial torsion subsheaf then the same is true of a dense set of closed fibers.

To finish the proof that $T$ and $F$ are open, it therefore suffices to prove the following: if $S$ is the spectrum of a complete dvr with closed point $s$ and $\mathscr{F}$ is an $S$-flat coherent sheaf on $X_{S}$, then $\mathscr{F}$ has torsion (resp. torsion free) fibers if and only if $\mathscr{F}_{S}$ is torsion (resp. torsion free). If $\mathscr{F}_{S}$ is torsion then it has support of dimension less than the dimension of $X$, and by upper semicontinuity the same is true of the generic fiber, establishing the result for $T$.

To establish the result for $F$, it suffices to show that if the generic fiber $\mathscr{F}_{\eta}$ contains a torsion subsheaf then so does the closed fiber $\mathscr{F}_{s}$. Suppose $\mathscr{G} \subset \mathscr{F}_{\eta}$ is a torsion subsheaf of the generic fiber. The closure $Z$ of the support of $\mathscr{G}$ in $X$ is $S$-flat, hence has closed fiber of dimension strictly smaller than the dimension of $X$. Let $\overline{\mathscr{G}} \subset \mathscr{F}$ be the subsheaf of sections with support in $Z$; it is the direct limit of the coherent subsheaves $\mathscr{H}_{o m_{O_{X}}}\left(\mathscr{O}_{X} / \mathscr{I}_{Z}^{n}, \mathscr{F}\right)$ of $\mathscr{F}$ and is thus coherent (as $\mathscr{F}$ is itself coherent). If $\sigma$ is a local section of $\mathscr{F}$ over an open $U \subset X_{S}$ such that $t^{n} \sigma$ has support in $Z$ then $\sigma$ itself must have support in $Z$. To see this, it suffices to prove that if $u \in U \backslash Z$ then the germ of $\sigma$ at $u$ is trivial. But the stalk $\mathscr{F}_{u}$ is still $S$-flat, so $t$ acts faithfully, and therefore if $t^{n} \sigma_{u}=0 \in \mathscr{F} u$ then we must have $\sigma_{u}=0$.

As a consequence, the quotient $\mathscr{Q}:=\mathscr{F} / \overline{\mathscr{G}}$ is itself $S$-flat. It follows that $\mathscr{G}_{s} \rightarrow \mathscr{F}_{S}$ is injective, yielding a torsion subsheaf of the closed fiber $\mathscr{F}_{S}$, as desired.

Proof that Example A.4(2) defines an open stack of torsion theories. To show that the condition that every Harder-Narasimhan factor has slope at most $\beta \cdot \omega$ is open, we will 
once again show that the locus is constructible and stable under generization. More precisely, let $\mathscr{F}$ be a flat family of quasi-coherent sheaves of finite presentation on $X \times B$ with torsion free fibers. We will show that the set of points $U \subset B$ over which the fibers of $\mathscr{F}$ are in $F$ is open. As above, by Grothendieck's theory of limits we may assume that $B$ is of finite type over $k$.

To show that $U$ is constructible, we may assume that $B$ is reduced and irreducible, and we wish to show that $U$ contains the generic point if and only if it contains an open subset of points. This follows from the fact that the slope is constant in a flat family and the existence of the relative Harder-Narasimhan filtration over a dense open subscheme of $B$ (Theorem 2.3.2 of [6]).

To show that $U$ is stable under generization, let $R$ be a discrete valuation $k$-algebra and $\mathscr{F}$ a flat family of torsion free coherent sheaves on $X \otimes R$ such that the closed fiber $\mathscr{F}_{s}$ is in $F$. The maximal destabilizing subsheaf $\mathscr{G}_{\eta} \subset \mathscr{F}_{\eta}$ on the generic fiber extends to a coherent subsheaf $\mathscr{G} \subset \mathscr{F}$ such that $\mathscr{F} / \mathscr{G}$ is $R$-flat. It follows that the closed fiber $\mathscr{G}_{S}$ gives a subsheaf of $\mathscr{F}_{S}$ whose slope must be at most $\beta \cdot \omega$. Since the slope is constant in a flat family, we see that $\mu\left(\mathscr{G}_{\eta}\right) \leq \beta \cdot \omega$, as desired. (That this passes to the geometric generic fiber follows from the compatibility of the Harder-Narasimhan filtration of $\mathscr{F}_{\eta}$ with extension of the base field, Theorem 1.3.7 of [6].)

The proof that $T$ is open is similar, but with an extra complication due to the presence of the torsion subsheaf. The point is that in both the proof of constructibility and stability under generization, one can assume that the torsion subsheaves form a flat subfamily of $\mathscr{F}$. Thus, one immediately reduces to showing that for a torsion free family $\mathscr{F}$, the locus over which all Harder-Narasimhan factors have slope strictly greater than $\beta \cdot \omega$ is open. One can again use the existence of the relative Harder-Narasimhan filtration to get constructibility.

Stability under generization can be proven by induction on the rank as follows. Note that if the Harder-Narasimhan factors have slope larger than $\beta \cdot \omega$, then $\mu\left(\mathscr{F}_{s}\right)>\beta \cdot \omega$. Thus, if $\mathscr{F}$ has semistable generic fiber then $\mathscr{F}_{\eta}$ must be in $T$ when $\mathscr{F}_{s}$ is. On the other hand, if $\mathscr{F}_{\eta}$ is not semistable, then there is a flat subfamily $\mathscr{G} \subset \mathscr{F}$ which agrees with the maximal destabilizing subsheaf on the generic fiber. On the other hand, $\mu\left(\mathscr{G}_{\eta}\right) \geq$ $\mu\left(\mathscr{F}_{\eta}\right)=\mu\left(\mathscr{F}_{s}\right)>\beta \cdot \omega$. The quotient $\mathscr{F} / \mathscr{G}$ is still flat, and the closed fiber is a quotient of $\mathscr{F}_{s}$. Since $T_{S}$ is closed under the formation of quotients, we conclude by induction that $\mathscr{F}_{\eta} / \mathscr{G}_{\eta}$ is in $T_{\eta}$, whence $\mathscr{F}_{\eta}$ is in $T_{\eta}$.

The formation of the derived category yields a fibered category $\mathcal{D} \rightarrow S$ which over $B \rightarrow S$ takes the value $D\left(\mathcal{A}_{B}\right)$. The fibered category structure comes from the derived pullback functors (and their natural functorialities). The substack $\mathcal{A}_{p f}$ gives rise to a substack $\mathcal{D}_{p f}$ by taking $\left(\mathcal{D}_{p f}\right)_{B}$ to be the subtriangulated category of $D\left(\mathcal{A}_{B}\right)$ generated by complexes with entries in $\left(\mathcal{A}_{p f}\right)_{B}$. (Note that the subtriangulated category generated by the same procedure by $\mathcal{A}_{p f}^{p}$ is not the same, although it does agree on fiber categories over field-valued points of $S$.)

Recall that a complex $E$ on $X$ is relatively perfect if for every pair of affine open subschemes Spec $A$ of $S$ and Spec $B$ of $X$ such that Spec $B$ maps into Spec $A$ under the structural morphism $X \rightarrow S$, the complex $\left.E\right|_{\operatorname{Spec} B}$ is quasi-isomorphic to a bounded complex 
of $A$-flat coherent $B$-modules. The complex $E$ is universally gluable if for every geometric point $\bar{s} \rightarrow S$ and every $i>0, \operatorname{Ext}_{X_{\bar{s}}}^{i}\left(E_{\bar{s}}, E_{\bar{s}}\right)=0$. Note that if $E$ is in the heart of a sheaf of $t$-structures on $X$, then it must be universally gluable. The sub-fibered category of $\mathcal{D}$ formed by relatively perfect universally gluable complexes is denoted $\mathscr{D}_{\text {pug }}(X / S)$. It is a standard result (Corollaire 2.1.23 of [2] or Theorem 2.1.9 of [1]) that $\mathscr{D}_{\text {pug }}(X / S)$ is a stack on the fpqc topology on the category of $S$-schemes. We recall the main theorem of [8].

Theorem A.5. The fibered category $\mathscr{D}_{\text {pug }}(X / S) \rightarrow S$ is an Artin stack locally of finite presentation.

This is the Mother of All Moduli Spaces: it contains the hearts of all of the sheaves of $t$ structures on $X$. In our case, we can make this precise as follows. Given a stack of torsion theories $(T, F)$, we can define a substack $\mathscr{D}_{(T, F)}(X / S)$ of $\mathscr{D}_{\text {pug }}(X / S)$ corresponding to the family of hearts of the tilting with respect to the torsion theory.

Definition A.6. Given a stack of torsion theories $(T, F)$, the stack of tilted hearts with respect to $(T, F)$ is the stack $\mathscr{D}_{(T, F)}(X / S)$ whose objects over $B \rightarrow S$ are objects $\mathscr{C}$ of $\mathscr{D}_{\text {pug }}(X / S)_{B}$ such that for every geometric point $s \rightarrow B$, the derived pullback $\left.\mathscr{C}\right|_{S} ^{\mathbf{L}} \in$ $D\left(X_{S}\right)$ is in the heart of the tilting with respect to the torsion theory $\left(T_{S}, F_{S}\right)$, i.e., it has cohomology only in degrees -1 and 0 with $\mathscr{H}^{-1}(E) \in F_{S}$ and $\mathscr{H}^{0}(E) \in T_{s}$.

Remark A.7. Using Lemma A.3, we see that when $B=\operatorname{Spec} K$ is a point, an object of $\mathscr{D}_{(T, F)}(X / S)_{\text {Spec } K}$ is precisely a perfect complex on $X_{K}$ in the heart of the usual tilting with respect to the $t$-structure given by $T_{K}$ and $F_{K}$. In other words, it is equivalent to check the property of Definition A.6 on spectra of residue fields of $B$ rather than geometric points, if desired.

The main result of this appendix is the following.

Theorem A.8. If $(T, F)$ is an open stack of torsion theories then $\mathscr{D}_{(T, F)}(X / S)$ is an open substack of $\mathscr{D}_{\text {pug }}(X / S)$.

Proof. We will show something a priori more general: given an affine scheme $B \rightarrow S$ and a relatively perfect complex $E$ on $X \times_{S} B$, there is an open subscheme $U \subset B$ parametrizing fibers in the heart of the tilting with respect to $(T, F)$. More precisely, we will show that there is an open subscheme $U$ such that for an affine scheme $b$ : Spec $A \rightarrow B$, the derived base change $E_{\operatorname{Spec} A}$ is in $\mathscr{D}_{(T, F)}(X / S)_{\operatorname{Spec} A}$ if and only if $b$ factors through $U$. By Definition A.6 and Remark A.7, it is enough to prove this when $A$ is a residue field of $B$.

To proceed, we first note that by 2.2.1 of [8] and 8.10.5 of [5] we may assume $B$ is Noetherian. Let $U \subset B$ be the subset parametrizing points over which the fiber of $E$ is in $\mathscr{D}_{(T, F)}$. To show that $U$ is open, it suffices (yet again!) to show that $U$ is constructible and stable under generization. By 2.1.3 and 2.1.4 of [8], the locus in $B$ over which the cohomology of the geometric fibers of $E$ is concentrated in degrees -1 and 0 is open. Thus, we may assume from the start that $\mathscr{H}^{i}(E)=0$ unless $i \in\{-1,0\}$. 
As in the proof that Example A.4(1) is an open stack of torsion theories, we know that (since $B$ is Noetherian) to show $U$ is constructible it suffices to assume that $B$ is reduced and irreducible, and then we wish to show that the generic point of $B$ is in $U$ if and only if an open subset of points is contained in $U$. By generic flatness, we may then shrink $B$ until the (coherent) cohomology sheaves $\mathscr{H}^{0}(E)$ and $\mathscr{H}^{-1}(E)$ are $B$-flat. It now follows from the Tor spectral sequence that the formation of $\mathscr{H}^{0}$ and $\mathscr{H}^{-1}$ are compatible with arbitrary base change on $B$. Since $T$ and $F$ are open, we see that $U$ must be open. Since we shrank $B$ to begin this part of the argument, we conclude only that $U$ is constructible (i.e., it contains a dense open if and only if it contains the generic point).

To show that $U$ is stable under generization, we may assume that $B=\operatorname{Spec} R$ is the spectrum of a discrete valution ring and that the special fiber is in $\mathscr{D}_{(T, F)}$. Write $b$ for the closed point of $B$ and $\eta$ for the open point. Let $t \in R$ be a uniformizer. Since $E_{b}$ has no cohomology of degree below -1 , the long exact sequence in cohomology associated to the triangle given by (derived-) tensoring $E$ with $0 \rightarrow \mathscr{O}_{X} \rightarrow \mathscr{O}_{X} \rightarrow \mathscr{O}_{X} \otimes_{R} \kappa(b) \rightarrow 0$ yields an exact sequence

$$
0 \rightarrow \mathscr{H}^{-1}(E) \stackrel{t}{\rightarrow} \mathscr{H}^{-1}(E) \rightarrow \mathscr{H}^{-1}\left(E_{b}\right) \rightarrow \mathscr{H}^{0}(E) \stackrel{t}{\rightarrow} \mathscr{H}^{0}(E) \rightarrow \mathscr{H}^{0}\left(E_{b}\right) \rightarrow 0
$$

where the indicated arrows are multiplication by $t$. We see immediately that $\mathscr{H}^{0}\left(E_{b}\right)=$ $\mathscr{H}^{0}(E) \otimes \kappa(b)$; moreover, $T$ is closed under the formation of quotients, so any quotient of $\mathscr{H}^{0}(E) \otimes \kappa(b)$ lies in $T_{b}$. Dividing out $\mathscr{H}^{0}(E)$ by its associated submodules lying over $b$ yields an $R$-flat quotient sheaf $\mathscr{Q}$ with generic fiber $\mathscr{H}^{0}\left(E_{\eta}\right)$ and such that $\mathscr{Q}_{b}$ is a quotient of $\mathscr{H}^{0}\left(E_{b}\right)$. Thus, $\mathscr{Q}_{b}$ lies in $T_{b}$, whence the generic fiber $\mathscr{H}^{0}\left(E_{\eta}\right)$ must lie in $T_{\eta}$, as $T$ is an open substack of the stack of flat families of coherent sheaves. To prove that $\mathscr{H}^{-1}\left(E_{\eta}\right) \in F_{\eta}$ is somewhat simpler. It follows from the exact sequence that $\mathscr{H}^{-1}(E)$ is flat over $R$; since $F$ is closed under subobjects and $\mathscr{H}^{-1}\left(E_{b}\right) \in F_{b}$, the openness of $F$ (which is a substack of the stack of flat families) shows that $\mathscr{H}^{-1}(E) \in F_{B}$. Thus, $\mathscr{H}^{-1}\left(E_{\eta}\right) \in F_{\eta}$, as required.

Corollary A.9. The fibered category $\mathscr{D}_{(T, F)}(X / S) \rightarrow S$ is an Artin stack locally of finite presentation.

\section{References}

[1] Abramovich, D., Polishchuk, A.: Sheaves of $t$-structures and valuative criteria for stable complexes. J. Reine Angew. Math. 590, 89-130 (2006) Z Zbl 1093.14026 MR 2208130

[2] Beĭlinson, A. A., Bernstein, J., Deligne, P.: Faisceaux pervers. In: Analysis and Topology on Singular Spaces, I (Luminy, 1981), Astérisque 100, 5-171 (1982) Zbl 0536.14011 MR 0751966

[3] Bridgeland, T.: Stability conditions on K3 surfaces. Duke Math. J. 141, 241-291 (2008) Zbl 1138.14022 MR 2376815

[4] Grothendieck, A.: Éléments de géométrie algébrique. III. Étude cohomologique des faisceaux cohérents. I. Inst. Hautes Études Sci. Publ. Math. 11, 167 pp. (1961) MR 0217085 
[5] Grothendieck, A.: Éléments de géométrie algébrique. IV. Étude locale des schémas et des morphismes de schémas. III. Inst. Hautes Études Sci. Publ. Math. 28, 255 pp. (1966) Zbl 0144.19904 MR 0217086

[6] Huybrechts, D., Lehn, M.: The Geometry of Moduli Spaces of Sheaves. Aspects Math. E31, Vieweg, Braunschweig (1997) Zbl 0872.14002 MR 1450870

[7] Laumon, G., Moret-Bailly, L.: Champs algébriques. Springer, Berlin (2000) Zbl 0945.14005 MR 1771927

[8] Lieblich, M.: Moduli of complexes on a proper morphism. J. Algebraic Geom. 15, 175-206 (2006) Zbl 1085.14015 MR 2177199

[9] Lieblich, M.: Remarks on the stack of coherent algebras. Int. Math. Res. Notices 2006, art. ID 75273, 12 pp. Zbl 1108.14003 MR 2233719 